\title{
Scaling and Similitude in Single Nozzle Supersonic Retropropulsion Aerodynamics Interference
}

\author{
Ashley M. Korzun ${ }^{1}$ \\ NASA Langley Research Center, Hampton, VA, 23681, USA \\ Louis A. Cassel ${ }^{2}$ \\ LZ Technology, 1110 NASA Parkway. Houston, TX, 77058, USA
}

\begin{abstract}
Retropropulsion, or the firing of rocket engines or motors into the direction of flight, is a method of spacecraft deceleration and soft landing that dates back to the early 1960s. Current conceptual designs for landing humans on the surface of Mars require supersonic retropropulsion, or initiation of retropropulsion at supersonic freestream conditions, as part of an extended powered descent phase of flight. The objective of this work is to identify the design parameters and flow condition bounds for self-similar behavior of powered descent aerodynamic interference in relevant flight environments. In applications of sub-scale test data, an "unknown" uncertainty lies in scaling to and from full-scale environments and systems. The issue of scaling for the opposing flows characteristic of powered descent is the focus of the following analysis, using data from wind tunnel testing of configurations with a single, central nozzle as a point of departure.
\end{abstract}

\section{Nomenclature}

$\begin{array}{ll}A & =\text { area, } \mathrm{m}^{2} \\ a & =\text { speed of sound, } \mathrm{m} / \mathrm{s} \\ C_{p} & =\text { pressure coefficient } \\ C_{T} & =\text { aerodynamics thrust coefficient } \\ C_{\tau} & =\text { propulsion thrust coefficient } \\ c_{p} & =\text { specific heat capacity, } \mathrm{J} / \mathrm{kg}-\mathrm{K} \\ D & =\text { diameter, } \mathrm{m} \\ H & =\text { shock height, } \mathrm{m} \\ h & =\text { specific enthalpy, } \mathrm{J} / \mathrm{kg} \\ M & =\text { Mach number } \\ M W & =\text { molecular weight, } \mathrm{kg} / \mathrm{kmol} \\ p & =\text { pressure, Pa } \\ q & =\text { dynamic pressure, } \mathrm{Pa} \\ R & =\text { radius, } \mathrm{m} \\ R e & =\text { Reynolds number }\end{array}$

$\begin{array}{ll}T & =\text { temperature, } \mathrm{K} \\ V & =\text { velocity, } \mathrm{m} / \mathrm{s} \\ \gamma & =\text { ratio of specific heats } \\ \rho & =\text { density, } \mathrm{kg} / \mathrm{m}^{3} \\ \tau & =\text { thrust, } \mathrm{N} \\ \text { subscripts } & \\ B & =\text { base } \\ e & =\text { nozzle exit condition } \\ j & =\text { jet flow condition } \\ r e f & =\text { reference area } \\ \infty & =\text { freestream condition } \\ 0 & =\text { stagnation condition } \\ 2 & =\text { post-shock condition } \\ * & =\text { nozzle throat condition }\end{array}$

\section{Introduction}

Retropropulsion, or the firing of rocket engines or motors into the direction of flight, is a method of spacecraft deceleration and soft landing that dates back to the early 1960s [1]. Use of retropropulsion during atmospheric flight has reemerged as a technology development objective over the past decade [1-6]. Two candidate applications have driven this resurgent interest:

- launch vehicle deceleration and maneuvering for landing and recovery [6], and

- entry, descent, and landing (EDL) of human-scale vehicles at Mars [1-3].

\footnotetext{
${ }^{1}$ Research Aerospace Engineer, Atmospheric Flight and Entry Systems Branch, Member AIAA.

${ }^{2}$ Technical Consultant, Senior Member AIAA.
} 
The most advanced launch vehicle application demonstrated is the SpaceX Falcon 9 first stage booster recovery, and applications to Mars EDL date back to the 1960s. A significant body of this historical work has been reviewed in the literature [1]. The objective of present NASA research is the EDL of payloads large enough to support human exploration on Mars.

System performance requirements for human Mars exploration include greater than an order of magnitude increase in payload mass over what can be delivered with legacy supersonic parachute technology (20+t vs. 1-2 $t)[2,3]$. Current conceptual designs require supersonic retropropulsion (SRP), or initiation of retropropulsion at supersonic freestream conditions, as part of an extended powered descent phase of flight [2]. Configurations of interest for human-scale Mars EDL include blunt entry capsule shapes similar to previous Mars landers, rather than the slender, low drag area configurations associated with launch vehicles. A principal technology development challenge for powered descent is the reduction of uncertainties in aerodynamics interference (AI) induced by the rocket engine exhaust plume interaction with the oncoming atmosphere. Uncertainties in SRP AI influence the design and selection of propulsion systems and vehicle configurations, as well as guidance and control strategies. These uncertainties must be better quantified to proceed confidently with design of systems most efficient for future Mars EDL implementation and, more importantly, more predictably reliable for crewed flight. Advances in technology since the earliest development of Mars exploration systems have changed the methods for reducing uncertainties in aerodynamics. Modern best practices balance a combination of computational fluid dynamics (CFD), ground testing, and flight testing, with a growing reliance on computational models with prediction uncertainties that have been verified by comparison with relevant experimental data.

The objective of this work is to identify the design parameters and flow condition bounds for self-similar behavior of SRP AI in relevant flight environments. It is postulated that design parameters and operating conditions that keep the interference flow within these bounds will minimize uncertainties in predictions of AI. In applications of subscale test data, an "unknown" uncertainty lies in scaling to and from full-scale environments and systems. The issue of scaling for the opposing flows characteristic of powered descent is the focus of the following analysis, using data from wind tunnel testing of configurations with a single, central nozzle as a point of departure. Conceptual landers have varying configurations of multiple nozzles; scaling and similarity for multiple nozzle configurations will be reported separately, as additional data become available. This work is specific to free-flight and does not consider effects arising from interaction with a landing surface.

This paper is organized as follows. Section III describes the plume interference flowfield and discusses candidate scaling parameters, the derivations of which are detailed in the Appendix. Section IV evaluates the proposed scaling parameters against existing historical and modern SRP wind tunnel test data.

\section{Plume Interference Flowfield and Challenges of Scaling}

The propulsive-aerodynamic interference associated with SRP is a highly complex fluid dynamics phenomena arising from the interaction between the oncoming freestream flow and opposing retropropulsion exhaust in free flight. This interaction is governed by elements dictating the vehicle-induced flowfield and by elements dictating the exhaust plume flowfield, illustrated in Fig. 1.

The vehicle-induced flowfield is characterized by the outer mold line (OML) of the vehicle, the local atmosphere and flight conditions along the descent trajectory, and the vehicle attitude. The exhaust plume flowfield is characterized by the engine operating conditions (plume physical and flow properties) and geometry, number of nozzles, and configuration or integration of the engines with the vehicle OML. The freestream may be supersonic, transonic, or subsonic, all of which are traversed as the vehicle decelerates along a descent trajectory.

The most general description of plume modes is that they are either under-expanded or over-expanded. In the former case, the nozzle exit pressure is higher than the local ambient pressure, and there are various shock structures within the plume, depending on the degree of under-expansion. In the latter, the nozzle exit pressure is lower than the local ambient pressure, and flow separation and shock interaction are present within the nozzle rather than the plume. In the analyses presented here, only supersonic freestream flows, zero-degree vehicle attitudes, and single nozzle configurations are considered to permit validation of the derived relationships with well-characterized ground test data.

Figure 1 shows characteristic flowfield features for SRP AI with a single, under-expanded nozzle. Figure $1 \mathrm{~b}$ identifies many of these same features in a Schlieren still image for $M_{\infty}=4.6$ and $C_{T}=2.0$, where $C_{T}$ is the aerodynamics thrust coefficient, equivalent to the thrust $(\tau)$ divided by the product of freestream dynamic pressure $\left(q_{\infty}\right)$ and aerodynamic reference area $\left(A_{r e f}\right)$. Scaling must consider the mechanisms governing these features within the composite flowfield. Some of these features are absent, or intrinsically different, in other modes of either the freestream or the exhaust plume. Consequently, focusing scaling on any one of them is likely to result in very limited, 
if any, range of applicability for the resulting parameters. All combinations of freestream and plume modes are characterized by strong viscous interaction phenomena and mixing shear layers between the plume and freestream flows. The mixing of the two is always a significant part of the aerodynamics. To summarize, what may be adequate similitude in each flow alone (i.e, Mach numbers, Reynolds numbers, geometric similitude of OML and nozzle, etc.) may not be sufficient to assure similitude in the extrapolation of the interference effects from sub-scale testing.

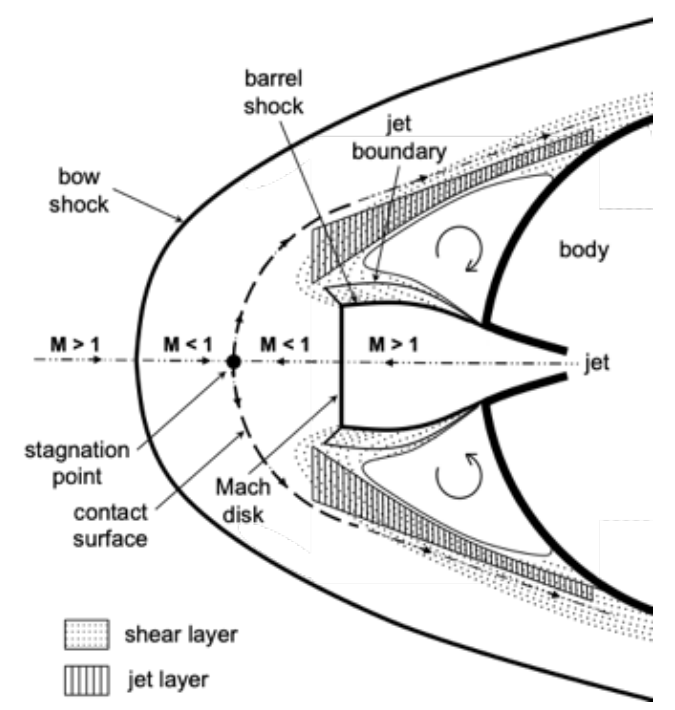

(a)

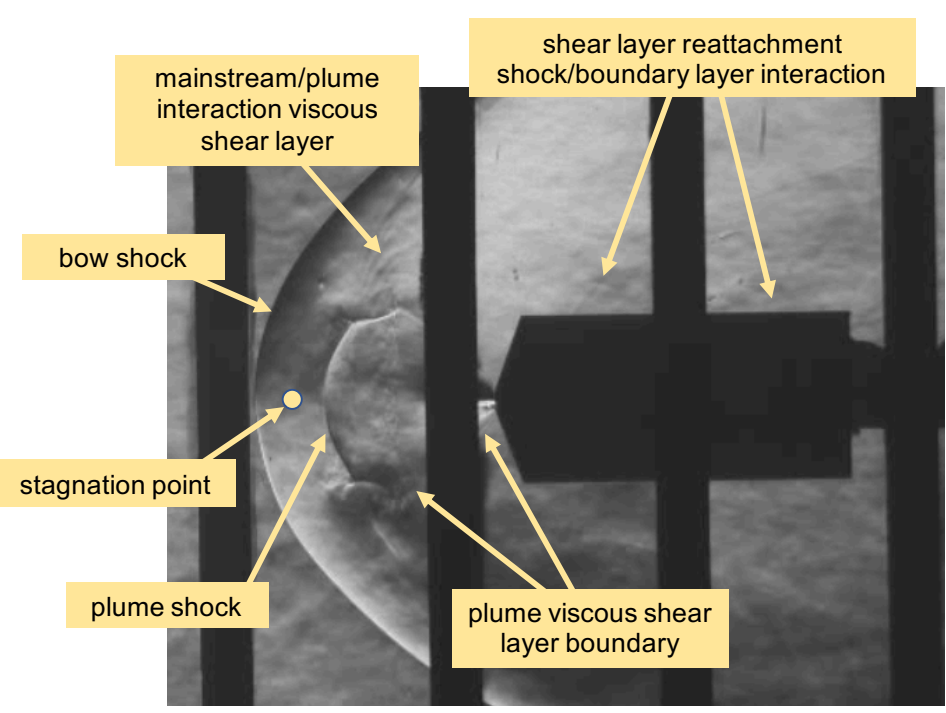

(b)

Fig. 1 Characteristic flow features in a typical retropropulsion - aerodynamic interaction [1,4].

SRP is generally implemented or conceptualized for very large flight systems. For example, the vehicles currently envisioned for human Mars exploration have axial dimensions upwards of $20 \mathrm{~m}$, and when combined with the extent of the AI flowfield during powered descent, may yield flow structures that alter the environments 10 s to 100 s of meters upstream of the vehicle [3]. For relevant testing in existing supersonic wind tunnels, these dimensions must be scaled down to tens of centimeters, or a two to three order of magnitude reduction in length scale. The significance of viscous interactions in SRP AI emphasizes the importance of Reynolds number simulation for extrapolation to flight. This simulation would require a two order of magnitude increase (over full-scale $R e_{\infty}$ ) in wind tunnel testing unit $R e$. The momentum conservation principles (discussed below) require thrust, relative to dynamic pressure, to be scaled with vehicle size. Since Reynolds number is proportional to dynamic pressure, the two requirements are contradictory.

With few exceptions, existing wind tunnels with aerodynamics testing capability were developed to simulate flight in the Earth's atmosphere. A select few, generally referred to as propulsion wind tunnels, have infrastructure compatible with injection of secondary gases at flow rates comparable to the freestream. A subset of these few are compatible with injection of secondary gases that contain products of combustion. Consequently, a requirement for scaling the physical properties of both the freestream and plume gases involved in Mars EDL SRP AI is added to the dimensional scaling requirement.

To guide the analyses described in this paper, an approach to scaling SRP is derived for environments consistent with those illustrated in Fig. 1. The derivation begins with the formation of a closed control volume around the vehicle and the SRP AI disturbance region, where the freestream and flow from the nozzles enter the volume, and the mixture flows out of the volume. The principles of conservation of mass, momentum, and energy are applied to produce integral equations for each relation. Those equations are reduced to similarity form, and the resulting parameters are then considered candidate scaling parameters for SRP AI. The details of this derivation are summarized in the Appendix. Table 1 summarizes these scaling parameters and the rationalizing equations used in their derivation.

Since the scaling model includes the interaction of the vehicle OML with the freestream and plume, it requires the scaling parameters of solid body aerodynamics to be observed (Table 1, row 1): freestream Mach number, freestream Reynolds number, and OML geometry. If the gases in the sub-scale testing are the same as in the full-scale objective, then approximations to mass flow and momentum conservation are achieved by matching the mass flow ratio and the 
ratio of thrust to the product of dynamic pressure and reference area, frequently referred to as the aerodynamics thrust coefficient, to differentiate it from the thrust coefficient common to nozzle design:

$$
C_{T}=\frac{\tau}{q_{\infty} A_{\text {ref }}}
$$

Table 1 Summary of scaling parameters for SRP AI

\begin{tabular}{|c|c|c|c|}
\hline Rationalizing Equations & Applications & Requirements & Scaling Parameter(s) \\
\hline \multirow{3}{*}{ Navier-Stokes } & \multirow{3}{*}{ All SRP geometries and flows } & Continuum gases & $M_{\infty}, R e_{\infty}$ \\
\hline & & Both plume and freestream gases & \multirow{2}{*}{$\begin{array}{l}\text { Geometric similitude in } \\
\text { freestream and plume flows }\end{array}$} \\
\hline & & Smooth, solid surfaces & \\
\hline \multirow[b]{2}{*}{ Momentum conservation } & \multirow{2}{*}{$\begin{array}{l}\text { Approximation of the scale of } \\
\text { AI region relative to vehicle }\end{array}$} & Meet above requirements & \multirow{2}{*}{$C_{T}=\frac{\tau}{q_{\infty} A_{\text {ref }}}$} \\
\hline & & $\begin{array}{l}\text { Similar freestream and plume gas } \\
\text { physical properties }\end{array}$ & \\
\hline \multirow[b]{2}{*}{ Mass conservation } & \multirow{2}{*}{$\begin{array}{l}\text { Approximation of the scale of } \\
\text { AI region relative to vehicle }\end{array}$} & Meet above requirements & \multirow{2}{*}{$\frac{\rho_{e} V_{e} A_{e}}{\rho_{\infty} V_{\infty} A_{\infty}}$} \\
\hline & & $\begin{array}{l}\text { Similar freestream and plume gas } \\
\text { physical properties }\end{array}$ & \\
\hline $\begin{array}{l}\text { Momentum and mass } \\
\text { conservation }\end{array}$ & $\begin{array}{l}\text { Scaling of AI flow from one } \\
\text { set of gases to another }\end{array}$ & $\begin{array}{l}\text { Meet above requirements except } \\
\text { that for similar gas properties }\end{array}$ & $\begin{array}{c}\gamma_{\infty}, \gamma_{e} \\
\frac{M W_{j} T_{0, \infty}}{M W_{\infty} T_{0, j}}\end{array}$ \\
\hline Energy conservation & Scaling of aeroheating effects & Meet above requirements & $\frac{c_{p, j} T_{0, j}}{c_{p, \infty} T_{0, \infty}}$ \\
\hline
\end{tabular}

The last two rows in Table 1 address scaling for the freestream and exhaust gas physical properties when the gases in the sub-scale experiment are different from those in the full-scale objective. Since the scaling model is postulated for perfect gas, steady-state flows, the scaling model does not differentiate between the temperature and molecular weight of gases. It also does not account for viscosity or thermal conductivity; the requirement to match $R e$ is taken as implicit in solid body force production, irrespective of whether SRP AI is present. The last row of Table 1 introduces energy conservation and the existence of heat transfer. The validation of the scaling parameters listed in Table 1 through evaluation against available ground test data for SRP AI is the focus of the remainder of this paper.

\section{Evaluation of SRP AI Scaling Parameters}

This section evaluates the derived candidate scaling parameters detailed in Table 1 against available data from both historical and modern wind tunnel testing. The data surveyed inform the effects of variations in nozzles, nozzle configurations, and aerodynamics parameters, primarily sourced from NASA wind tunnel testing conducted in the era of the Viking Mars lander development [1,7-10]. A pair of NASA wind tunnel tests in 2010 and 2011 expanded upon legacy efforts through parametric investigation of single and multiple nozzle configurations across a range of supersonic Mach numbers and thrust coefficients [4,5]. This most recent testing incorporated modern pressure instrumentation and examined a number of multiple nozzle arrangements and a range of flow conditions. The combination of these data indicates broad variations in plume interactions and resulting aerodynamic interference. These variations are on a geometric scale such that the resulting size of the interference region changes by multiples of its size under other conditions, with large variations of unsteady flow on the same scale. Both plume interactions and the resulting aerodynamic inference can be strongly dependent on elements of the vehicle and propulsion system configuration under a variety of flow conditions.

Most of the legacy testing was designed to explore AI phenomena, not scaling or links to a particular reference flight implementation. However, several of the tests incorporated variations in nozzle and/or model geometry. In contrast, the recent NASA testing was specifically designed to provide experimental data to be used for validation of computational fluid dynamics (CFD) codes and to re-establish the capability to investigate these interference effects through ground testing [4,5]. This testing also incorporated variations in nozzle (and therefore plume) configuration without replication of the historical references for multiple nozzle data. All of the experiments evaluated in this analysis employed gaseous air as the freestream fluid. Nearly all of the data were taken employing air as the plume 
gas. A limited number of legacy tests evaluated the effects of helium as the plume gas simulant over a limited range of conditions. Several of the experiments, including the last test series by NASA, employed a wide variation in angle of attack. The present data analysis addresses only the zero angle of attack condition, where the mean flow can be assumed to be axisymmetric. It is implicit in the proposed scaling model that similitude is provided irrespective of geometry or attitude relative to flow direction. The requirement is that the sub-scale tests duplicate, or nearly approximate, the full-scale attitudes and flow directions.

The following analyses are directed at evaluation of the large variations in the scale of the interference flow with variations in vehicle configuration (OML), nozzle configuration, exhaust plume flow conditions, and freestream flow conditions. Similitude is evaluated by whether or not the extent or size of the AI region scales with the parameters derived in the model. The objective is to identify ranges of blunt body configuration parameters and flow conditions over which the interference flow behavior approximates a self-similar change in physical scale as chamber pressure (e.g. nozzle total pressure) is varied, conditions equivalent to throttling. Since there are no variations in gas properties of either the freestream or plume gases in the database (except for the limited data employing helium as the plume gas simulant), only the first two rows in Table 1 are pertinent to this evaluation. The remaining rows of Table 1 require additional ground testing, with simulant gases other than high-pressure air, for evaluation.

The SRP AI flowfields documented with existing test data do not fall into a similar set. There appear to be one set with bow shock shapes clearly associated with blunt bodies. Another set of bow shock shapes appears to be associated with more slender configurations, even though the configuration from which the plume(s) originate is a blunt body. In many cases, there is a transition from one set of bow shock shapes to the other with only changes in the nozzle chamber pressure. These transitions have been observed for both single and multiple nozzle configurations. The analyses by Finley [9] and by Jarvinen and Adams [10] describe behavior of this phenomena in their data. As it is well documented that the nozzle expansion condition, and therefore the exhaust plume, can change dramatically with the ratio of nozzle chamber pressure-to-ambient pressure at the nozzle exit $\left(p_{0,2} / p_{e}\right)$, the hypothesis is made that these transitions are influenced by such changes in external pressure at the nozzle exit. Different evaluations of the phenomena in SRP differ principally in how the ambient pressure is characterized.

Because the AI obstructs the oncoming freestream flow, the ambient pressure at the nozzle exit is, in most cases, not equivalent to the freestream ambient pressure. Consequently, a better approximation of the external pressure at the nozzle exit is required to estimate the expansion mode of flow exiting the nozzle. For the highly under-expanded plume shown in Fig. 1, the mutual stagnation point, where the plume centerline velocity meets the freestream centerline velocity, appears near the termination of the expansion of the plume from the nozzle. Since it is downstream of the bow shock in the freestream, pressure there should approximate freestream post-shock stagnation pressure. A CFD solution of a similar flow (see [6]) confirms this approximation, as shown in Fig. 2.

The operative hypothesis is then that the plume would be in the under-expanded mode when the exit pressure exceeds the freestream post-shock stagnation pressure. This presents two hypotheses to be tested against both legacy and modern wind tunnel test data:

1. The size of the AI region will scale with momentum ratio (or the approximately equivalent $C_{T}$ ), if the plume is in a highly under-expanded mode. The measure of the disturbance size is the height of the interference flow bow shock above the nose of the vehicle, measured along the centerline.

2. The plume would be in an under-expanded mode if the exit pressure exceeds the freestream post-shock stagnation pressure.

Historical SRP wind tunnel tests explored a wide variety of axisymmetric configurations with simulated single nozzles on the centerline, examples of which are shown in Fig. 3. The configurations range from slender blunted cylinders [7] to more traditional blunt body aeroshells [4,5,8-11]. In some tests, nozzle dimensions and expansion ratios were also varied. In the figures that follow, nozzle expansion ratio will be identified by nozzle exit Mach number $\left(M_{e}\right)$, and nozzle dimensions will be identified by the ratio of model base diameter to nozzle throat diameter $\left(D_{B} / D^{*}\right)$. Documentation of historical testing is limited to descriptions and data recorded in the original test reports, which may lack complete information to fully define run conditions. Participants and their records are available for the more recent NASA testing, lending more confidence to interpretation of these data.

The primary flow feature common across all data is the bow shock, noted in Fig. 1, which defines the upstream boundary of the AI region for SRP. The shape of that shock and its position relative to the vehicle OML are unique characteristics associated with the interference of the two opposing flows. It is postulated that self-similar growth in the physical extent of this specific shape, as a function of a single parameter combining both flows and geometries, indicates that the parameter provides first-order similitude in extrapolation of the AI from a smaller to a larger geometric scale. The shock stand-off distance from the vehicle nose (stagnation point on the heatshield) is considered 
to be the characteristic dimension of the AI flowfield in this analysis. Normalized by the vehicle diameter, differences in this dimension are compared at constant values of the scaling length (or its square).

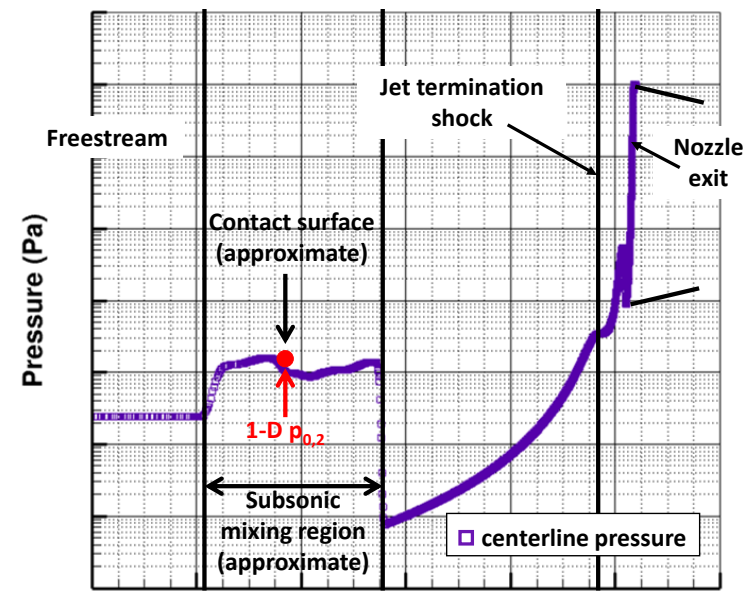

Fig. 2 Pressure along AI centerline [6].

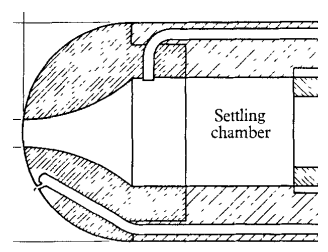

Finley [9]

$D_{B} / D^{*}=8$

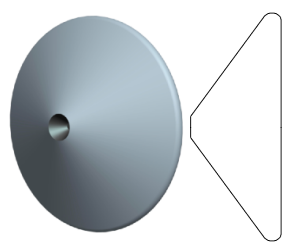

Jarvinen \& Adams [10] $D_{B} / D^{*}=30$

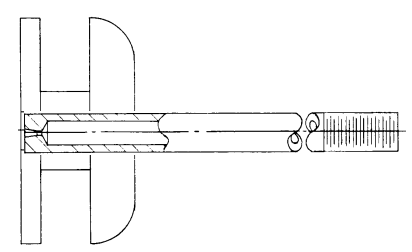

Romeo \& Sterrett [7] $D_{B} / D^{*}=10,58$

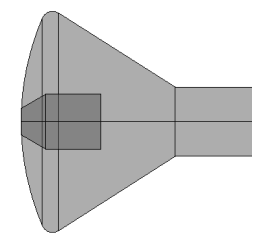

Daso [11]

$D_{B} / D^{*}=8,11$

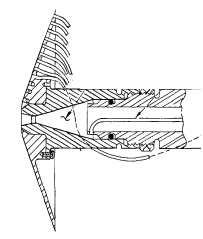

McGhee [8] $D_{B} / D^{*}=37$

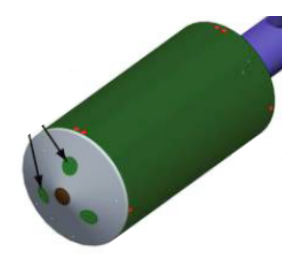

Berry et al. [4,5]

$D_{B} / D^{*}=20$

Fig. 3 Single nozzle model configurations.

Figure 4 shows a series of instantaneous Schlieren images with increasing $C_{T}$ for a single supersonic nozzle plume of air in an air freestream at $M_{\infty}=2.4$. Figure 5 shows the momentum ratio scaling and post-shock stagnation pressure criteria for the single (under-expanded) nozzle flow mode. These data are from the testing reported by Berry et al. [4,5] in 2010 and 2011. The relative similarity of the interference flows over the range of thrust coefficients tested (achieved by increasing nozzle chamber pressure $\left(p_{0, j}\right)$ ), is qualitatively confirmed from visual inspection of Fig. 4 , and quantitatively confirmed by the linear relationships with bow shock height in Fig. 5.

The linear correlation of normalized bow shock height with the square root of the momentum ratio is highly consistent with the scaling law relationship between the AI cross-sectional area and the momentum ratio. The distributions under the integrals on the right-hand side of the momentum ratio equation (see Eq. 11 in the Appendix) are likely to be very similar for this self-consistent data set, lending credence to the single-mode behavior of the plume (e.g. the plume remains highly under-expanded). The data in Fig. 5b confirm that the nozzle exit pressure exceeded the freestream post-shock stagnation pressure for data in Fig. 5a. They also indicate that the same pressure ratio scales the disturbance size in the same manner as the momentum ratio when the nozzle chamber pressure is the only parameter varied. 


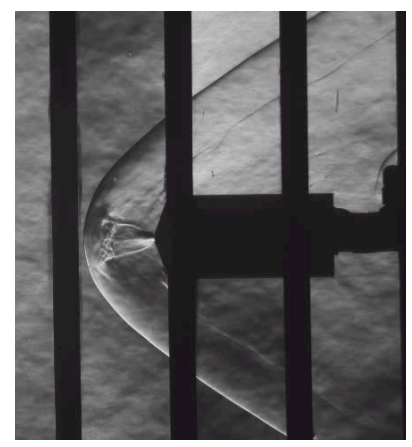

$C_{T}=0.5$

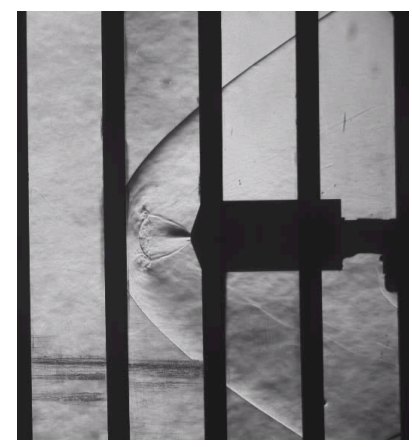

$C_{T}=1.0$

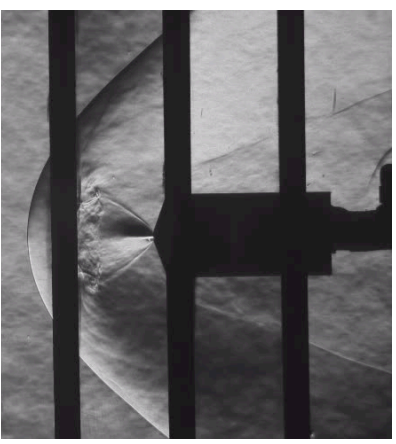

$C_{T}=2.0$

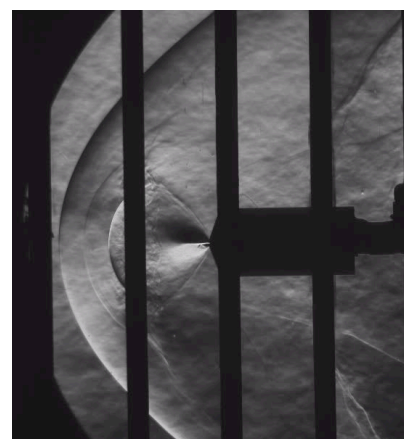

$C_{T}=4.0$

Fig. 4 Instantaneous Schlieren images of SRP AI for a single supersonic nozzle plume $\left(M_{\infty}=2.4\right)[4]$.

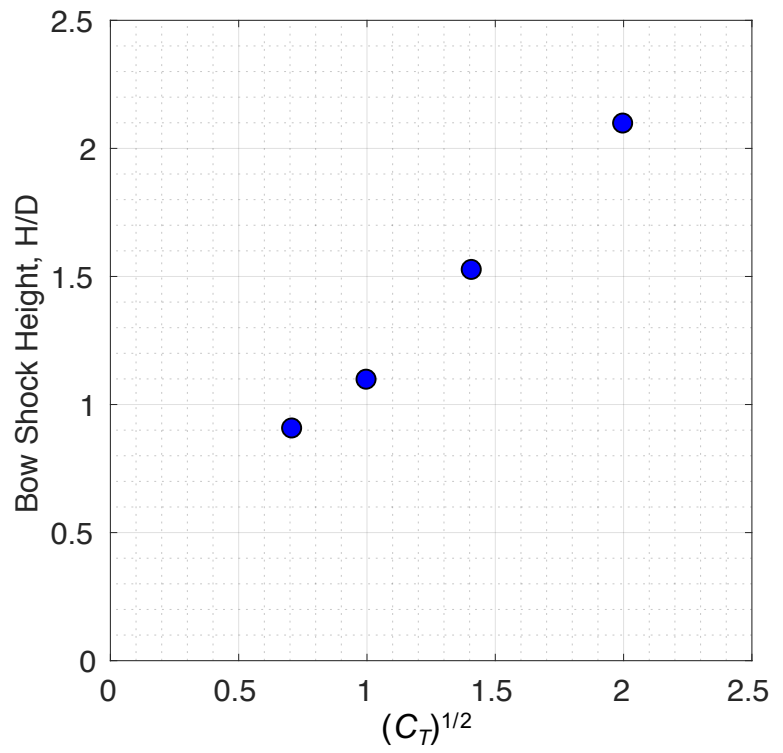

(a)

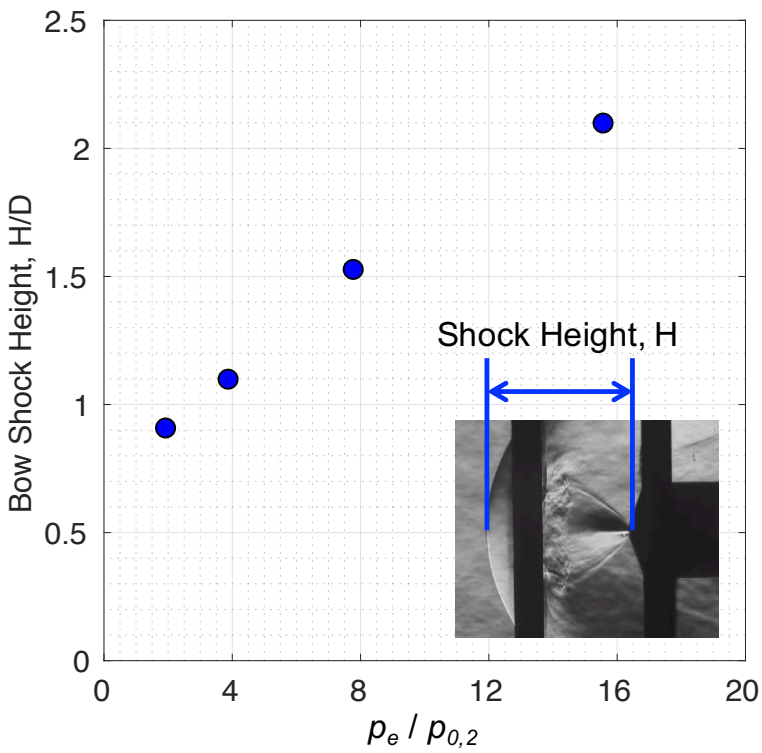

(b)

Fig. 5 Momentum ratio scaling of SRP AI flows shown in Fig. 4.

In Fig. 6, the correlations in Fig. 5 are extended in the range of both $C_{T}$ and $M_{\infty}$ with data from Jarvinen and Adams [10], which have a slightly different OML (see Fig. 4) and different Mach numbers in both the freestream and plume exhaust flows. Figure 6 reflects favorably on the correlation of momentum ratio with AI disturbance size and also supports the hypothesis that self-similarity exists for under-expanded nozzle flows when the ambient pressure at the nozzle exit exceeds the freestream post-shock stagnation pressure $\left(p_{e} / p_{0,2}>1\right)$. In the data from Jarvinen and Adams [10], an abrupt change is observed in the interference flow size for conditions near $C_{T}=2.0$ and $p_{e} / p_{0,2}$ exceeding unity, suggesting that the plume exhaust flow becomes under-expanded near these conditions. While it seems reasonable that the nozzle exhaust flow would be isolated from the freestream flow at some point with increasing nozzle chamber pressure, it is not obvious that the interference flow should become self-similar in behavior as chamber pressure (or more generally, momentum ratio) is increased beyond this threshold. The explanation is provided in Fig. 7 using the forebody pressure distribution as a function of $C_{T}$. By comparison with the indicated base pressure value, it is evident that the flow over the forebody separates when $C_{T}$ reaches 2.0 for these particular freestream conditions. From Figs. 6 and 7, the combination of the under-expanded exhaust plume (isolating the flow in the nozzle from the external AI) and flow separation over the forebody (stabilizing the AI flow structure in the shock layer) appear to constrain the overall AI region to self-similar growth with increasing $C_{T}$. This growth persists over the interval of $C_{T}$ shown in Fig. 6. 


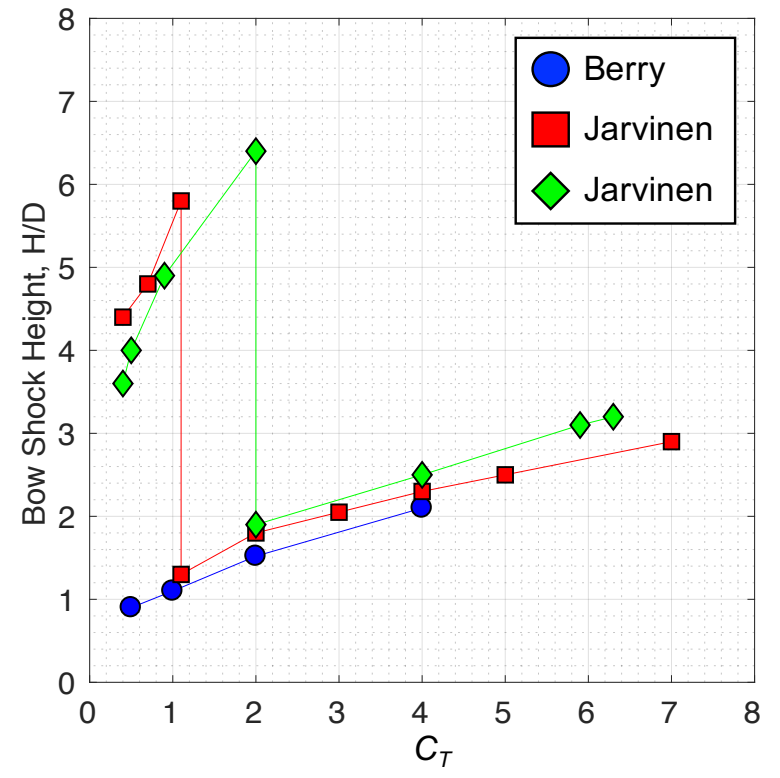

(a)

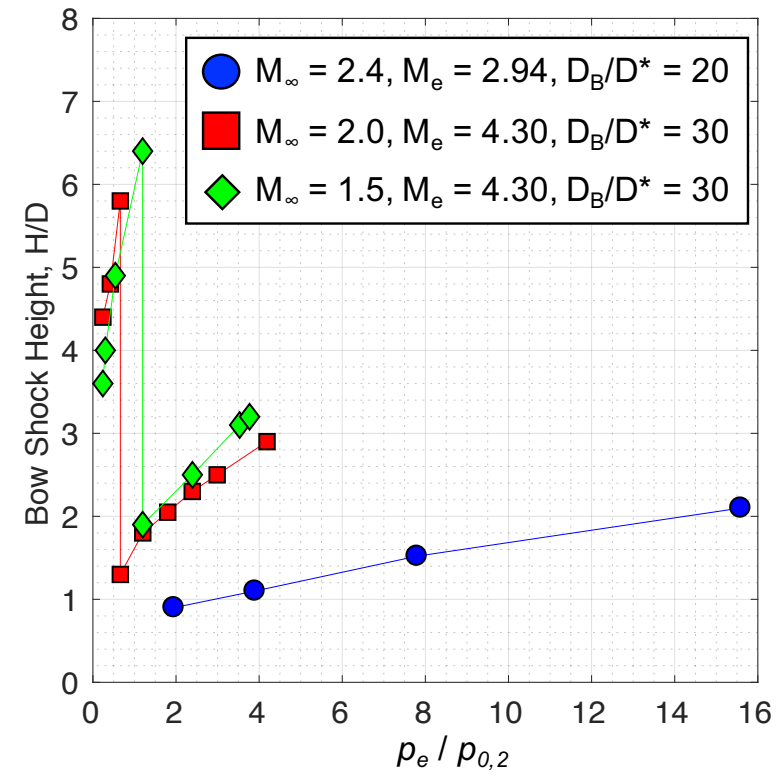

(b)

Fig. 6 Extension of momentum ratio correlation and discontinuous trends in AI size with increasing momentum ratio.

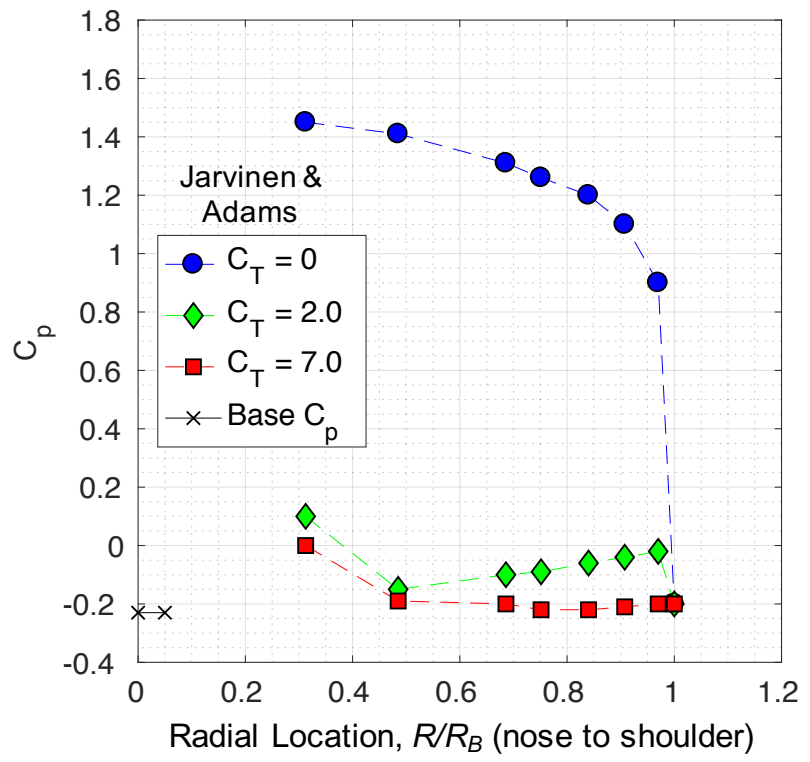

(a)

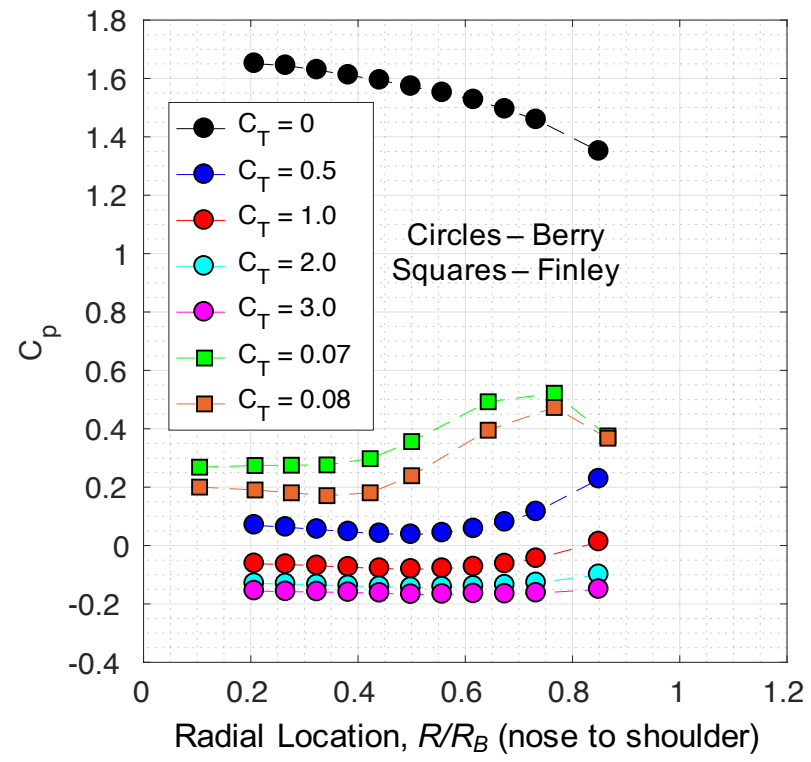

(b)

Fig. 7 Forebody $C_{p}$ distributions as a function of $C_{T}$ (data from $\left.[4,5,9,10]\right)$.

In Fig. 7, historical data reported by Finley [9] further corroborates the observation of the relationship between forebody flow separation and self-similarity in AI disturbance behavior with increasing $C_{T}$ at supersonic freestream conditions. With the addition of data from Finley [9], there are now differences between freestream and nozzle exit Mach numbers, as well as forebody shape (see Fig. 4), though all are still characteristically blunt bodies with a single, center nozzle and equivalent $D_{B} / D^{*}$. The trend in surface pressure with increasing $C_{T}$ is consistent across Berry [4,5], Jarvinen and Adams [10], and Finley [9].

Figure 8 adds data from other single nozzle configurations tested with air plumes exhausting into air freestreams to that shown previously in Fig. 6. With the inclusion of these data into the similitude evaluation, the correlation between momentum ratio and AI disturbance size becomes less apparent. Similitude in the interference flow is 
unlikely to be achieved without geometric similarity in the wind tunnel models, as differences arise, in part, from differences in model OML between datasets (see Fig. 4). The correlation of AI disturbance size with $C_{T}$, in Fig. 8a, remains credible at thrust coefficients exceeding unity, even with a larger variation in $M_{\infty}$ and OML. Conversely, the data in Fig. 8 demonstrate that the postulate of a single, similar inference flow mode is not held for $C_{T}<1$. In Fig. $8 \mathrm{~b}$, the postulate of $p_{e} / p_{0,2}$ indicating a mode change is tested with the additional data. Here, it appears that the ratio $p_{e} / p_{0,2}$ must exceed 3, not unity, to support the correlation of disturbance size to approximations that may be considered consistent with the quality and interpretation of the underlying historical data. However, the absence of correlation in AI disturbance size at values of $p_{e} / p_{0,2}$ approaching (or below) unity is reinforced.

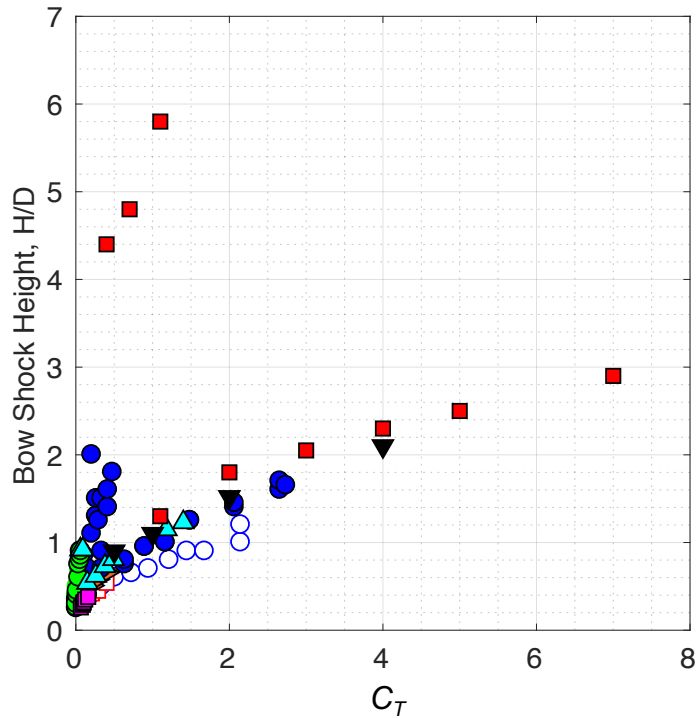

(a)

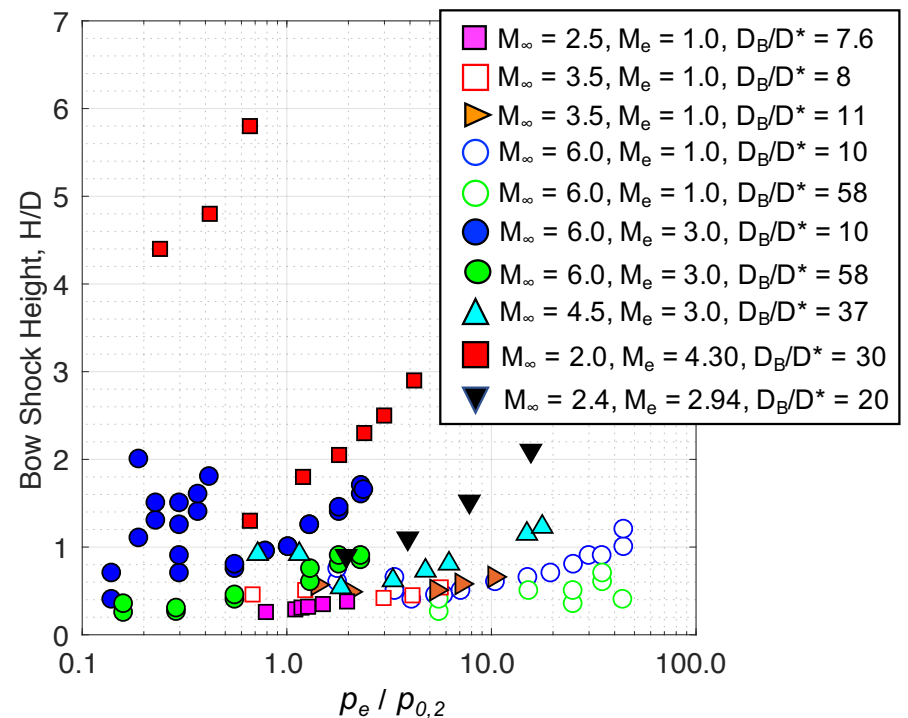

(b)

Fig. 8 Interference size with $C_{T}$ and $p_{e} / p_{0,2}$ for legacy and modern test data [4,5,7-11].

Much of the SRP wind tunnel testing to date has shown evidence of multiple modes of plume interference at lower values of momentum ratio and $p_{e} / p_{0,2}$. Data from Romeo and Sterrett [7], shown in Fig. 8 as data at $M_{\infty}=6.0$, provides insight into the relationship between AI disturbance (e.g. bow shock) size and the relative difference in size between the nozzle and body. This testing used the ratio between body diameter and nozzle throat diameter, $D_{B} / D^{*}$ (if $M_{e}$ is treated as an independent variable), to characterize this relationship. It should be expected that the $C_{T}$ correlation should contribute to scaling with the $D_{B} / D^{*}$. The nozzle design thrust coefficient, $c_{t}$, is defined by the nozzle expansion ratio and exhaust plume gas ratio of specific heats such that:

$$
c_{t}=\frac{\tau}{p_{0, j} A^{*}}
$$

The approximation of momentum ratio, using $c_{t}$, can then be written as:

$$
C_{T}=\frac{c_{t} p_{0, j} A^{*}}{q_{\infty} A_{\text {ref }}}
$$

From Eq. 3, it then follows:

$$
C_{T}=\left(\frac{c_{t} p_{0, j}}{q_{\infty}}\right)\left(\frac{1}{D_{B} / D^{*}}\right)^{2}
$$

When $C_{T}$ is written in this form (Eq. 4), the flow variables are now separate from those defining the geometry, allowing for identification of the comparative contributions of the two groupings in Eq. 4. It must be noted that, as in all aerodynamics, changes in geometry produce changes in the composite flowfield arising from the AI disturbance. If one of the variables defining the diameter ratio is changed independently, either the freestream or plume momentum is changed independently. In either case, the momentum ratio represented by $C_{T}$ is also changed. 
In some of their testing, Romeo and Sterrett [7] held the set of flow variables (the left-hand parentheses in Eq. 4) constant while changing the model body diameter. The influence of body diameter on the interference flow is illustrated by the Schlieren still images in Fig. 9, with ratios of body diameter-to-throat diameter of 9.7, 19, and 58. The flow variables are the same for each of the cases shown: $M_{\infty}=6$ air freestream and $M_{e}=3$ air nozzle flow at the same chamber pressure. The forebody shapes are also the same in each image in Fig. 9, yet the interference flows are clearly different.

$$
M_{\infty}=6.0, p_{e} / p_{0,2}=2.3, M_{e}=3
$$

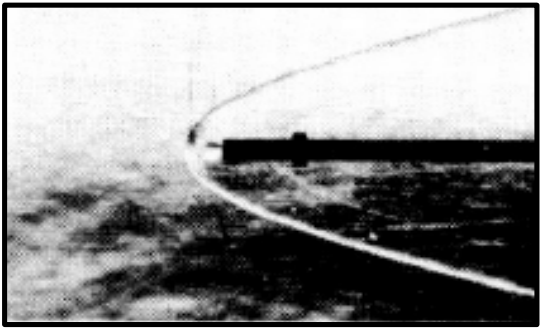

$D_{B} / D^{*}=9.7$

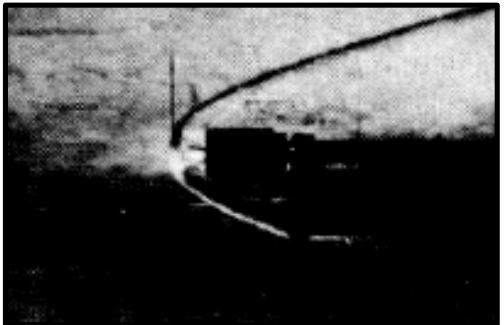

$D_{B} / D^{*}=19$

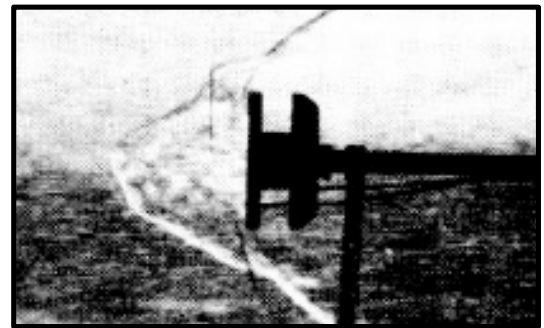

$D_{B} / D^{*}=58$

Fig. 9 Influence of configuration bluntness on interference flow [7].

Figure 10 shows schlieren images for two $D_{B} / D^{*}$ extremes of the configuration shown in Fig. 9. The upper series of images in Fig. 10 represents the smallest diameter tested. The bottom series of images in Fig. 10 represents the largest diameter tested. Similar to Fig. 9, all of the images in Fig. 10 are at the same $M_{\infty}=6$ condition; all variations are due to changes in body or nozzle geometry, or in-nozzle flow conditions. The latter are associated with variations in chamber pressure when the (ideal) exit Mach number is unchanged.
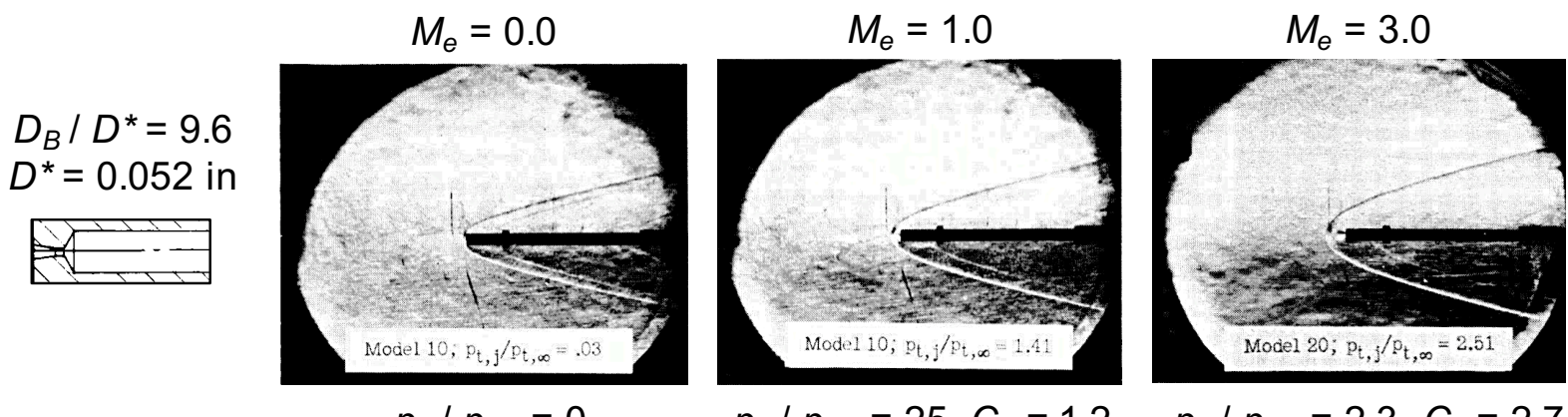

$$
p_{e} / p_{0,2}=0
$$
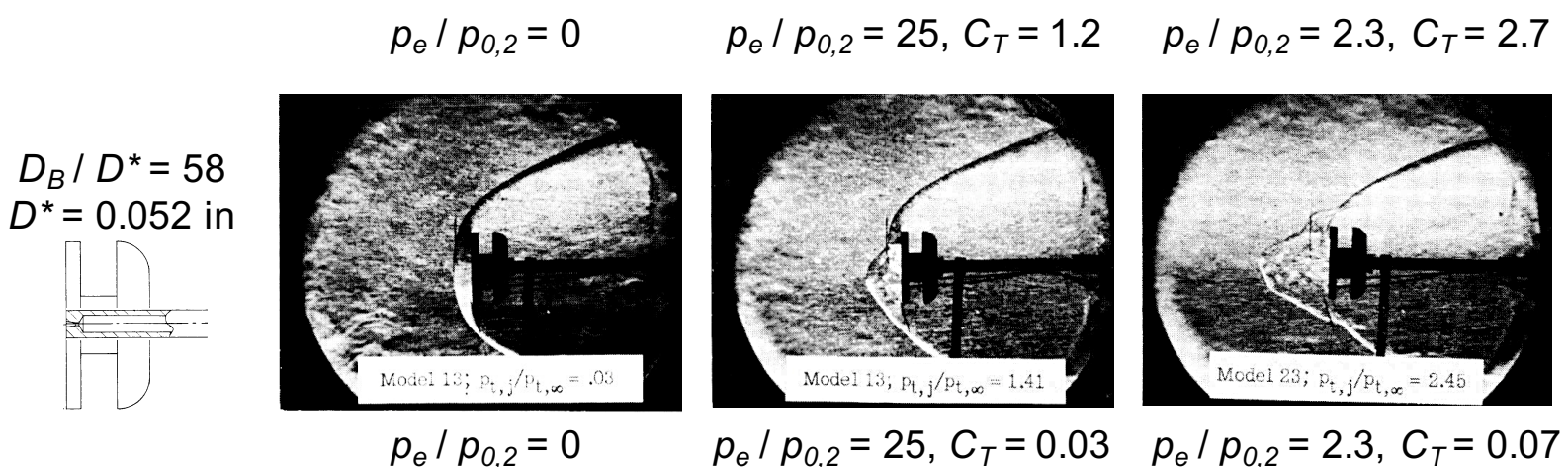

Fig. 10 Examples of variability in AI region shape with nozzle size and body diameter for $M_{\infty}=6$.

In both the upper and lower series of images in Fig. 10, the left-most Schlieren image shows the body-alone flowfield. Both bodies are flat faced cylinders, so the shock shapes associated with both should be the same (compared to the body) in the unpowered (no nozzle flow) cases. However, this similarity is at the body diameter scale. The nozzle exit diameters are the same in both models, and the Schlieren field of view is at the throat diameter scale, so the upper images are at $20 \%$ of the scale in the lower images. Consequently, at nozzle scale, the shock structure for 
the smaller $D_{B} / D^{*}$ (upper series) has the appearance of a slender body (or weak/oblique) shock. The shock structure associated with the larger value of $D_{B} / D^{*}$ (bottom series) has the appearance of a blunt body (or strong) shock. As a result, the scale of the plume, relative to that of the body, is much different for the two different $D_{B} / D^{*}$ ratios. The pressure distribution that the plume exhausts into, at nozzle scale is also then quite different for the two configurations in Fig. 10. While the centerlines for both models may see freestream stagnation pressure locally, it is unlikely that the average over the nozzle exits is the same for both geometries.

The center images in Fig. 10 illustrate the plume interference at the highest $p_{e} / p_{0,2}$ level tested. Unlike the other images in Figs. 9 and 10, these images show the plume for a sonic nozzle, rather than one expanded to $M_{e}=3$. The $C_{T}$ approximation of the momentum ratio is relatively low for the more slender body and vanishingly small for the more blunt body. The detached shock in the upper image appears to be displaced by the plume but retains the slender body shock shape (at the scale of the image). The detached shock in the lower image is also displaced but is significantly changed in shape. It no longer has the blunt body shock shape of the unpowered case, now resembling the shape of a shock for a spike protruding from a blunt body [12].

In the right-hand images in Fig. 10, $M_{e}$ is increased to 3, reducing $p_{e} / p_{0,2}$ but increasing $C_{T}$. Since plume gas properties are unchanged, exit velocity increases with exit Mach number. Here, the slender body shock displacement (upper image) is increased while the shape of the shock appears to be relatively unchanged. As momentum is added to the plume, the blunt body shock (lower image) is further displaced, and the shape is changed again, now resembling the shape of a shock for a conical protrusion from a blunt body [12].

The relationships between the parameters illustrated in Fig. 10 are brought out by the explicit equation for $C_{T}$ in terms of the nozzle thrust coefficient in the form:

$$
C_{T}=c_{t}\left(\frac{p_{0, j}}{p_{e}}\right)\left(\frac{p_{0,2}}{q_{\infty}}\right)\left(\frac{p_{e}}{p_{0,2}}\right) \frac{1}{\left(D_{B} / D^{*}\right)^{2}}
$$

This relationship is illustrated graphically with typical legacy wind tunnel data in Fig. 11. None of the data reviewed in these studies was from experiments directed at AI associated with launch vehicle SRP. However, those configurations typically tend toward low values of $D_{B} / D^{*}$ while atmospheric EDL configurations tend toward higher ratios.

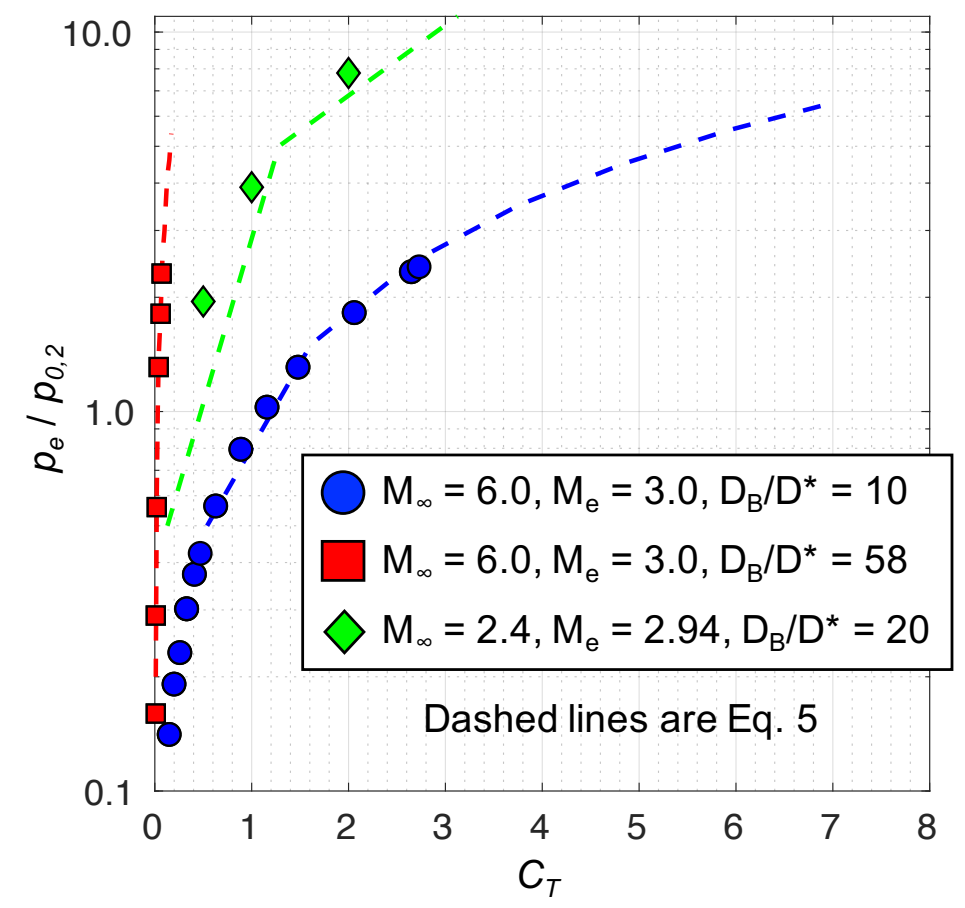

Fig. 11 Relationship between $p_{e} / p_{0,2}$ and $C_{T}\left(\gamma_{\infty}=\gamma_{e}=1.4\right)$.

It is evident from Fig. 11 that large values of $D_{B} / D^{*}$ require larger values of $p_{e} / p_{0,2}$ to achieve even small gains in retropropulsion effectiveness (compared to aerodynamic drag). In addition, if the postulate that the interference 
flow is not self-similar for $p_{e} / p_{0,2}$ values much smaller than unity is demonstrated to be valid, a substantial variability in these interference flows can be expected for larger ratios of body-to-nozzle diameter. The inverse also appears to be the case. When the body-to-nozzle diameter ratios are very small, greater retropropulsion effectiveness can be expected at relatively low values of $p_{e} / p_{0,2}$. These conditions are expected to yield interference flows that are selfsimilar and therefore consistent in behavior. In Fig. 12, an inference of these two modes is evident when the data shown previously in Fig. 8a are screened by the postulate that the interference flows are self-similar when $p_{e} / p_{0,2}$ exceeds unity. The data for $D_{B} / D^{*}=58$ appear to correlate differently than the data for the lower ratios. In the data for lower ratios, there appears to be a trend to larger disturbance sizes for larger values of $D_{B} / D^{*}$.

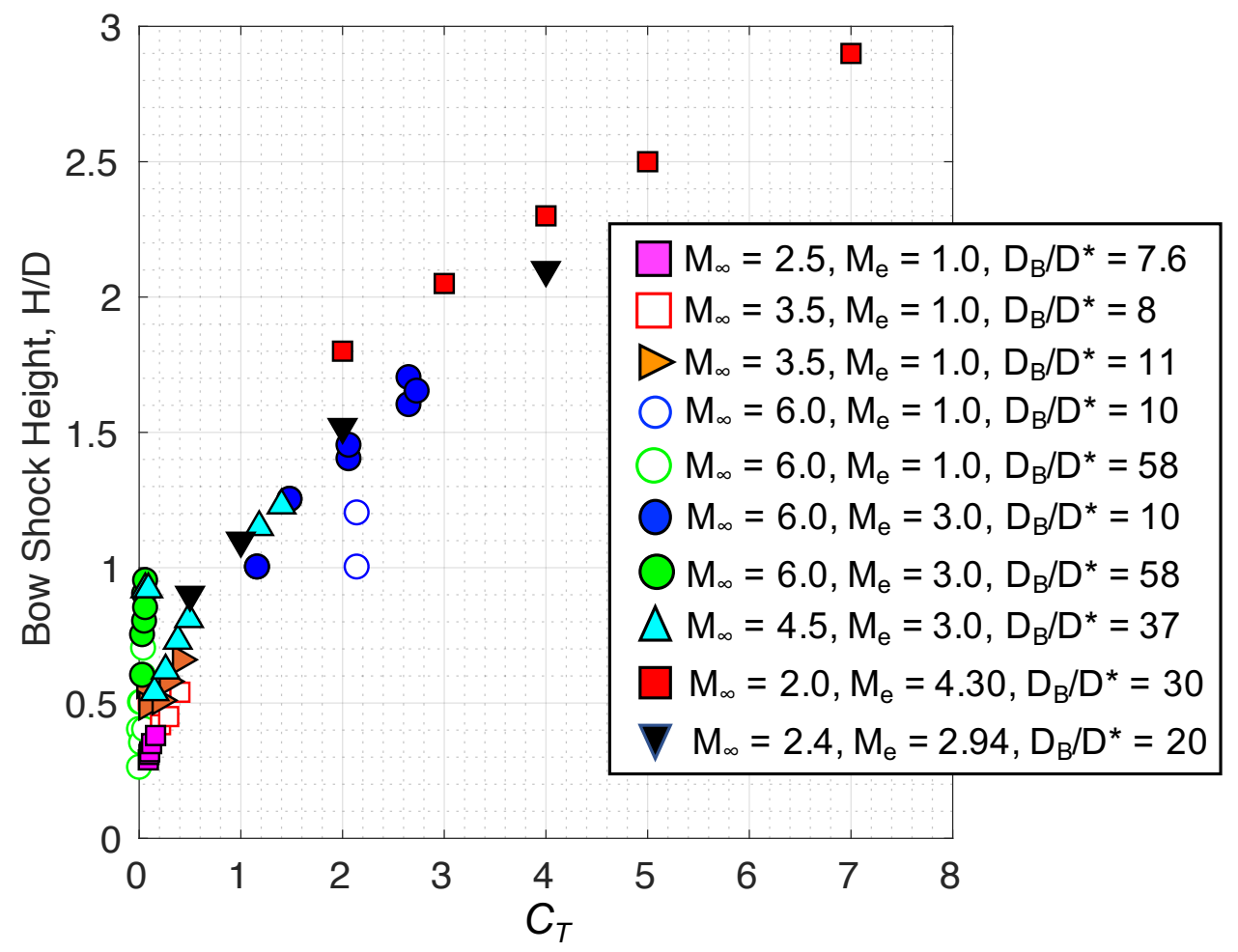

Fig. 12 Disturbance size correlation when plume is under-expanded $\left(p_{e} / p_{0,2}>1\right)$.

In the literature, Finley [9] recognized three modes of interference, testing models with various ratios of body-tonozzle diameter. In the data are surface pressure distributions on a spherical model with near equal flow conditions in both the nozzle and the freestream but $D_{B} / D^{*}$ values of 7.6 and 33. These data $\left(M_{\infty}=2.5\right)$, shown in Fig. 13, also conform to the criteria that $p_{e} / p_{0,2}$ exceed unity. In Fig. 13, all data are for $M_{e}=1$ (sonic nozzle), similar to a portion of the Romeo and Sterrett data $\left(M_{\infty}=6\right)$ [7] in Fig. 12. The latter data appear to be in family with the data from supersonic nozzles when $p_{e} / p_{0,2}$ is considered. Between Figs. 12 and 13, the data indicate that self-similar conditions for disturbance size growth are not present for large values of $D_{B} / D^{*}$ at low values of $C_{T}$.

As described in Section II, the objective of the work described here is to reduce uncertainties in scaling SRP aerodynamics interference from wind tunnel testing to Mars EDL scenarios. The evaluation of the SRP AI scaling described above has been limited to the finding self-similar growth in the size of the disturbance, defined by the mixing region in the vicinity of the plume, for air plumes exhausting into air freestreams. However, current projections for Mars EDL applications are that the exhaust plume will be the products of liquid oxygen/methane $\left(\mathrm{O}_{2} / \mathrm{CH}_{4}\right)$ combustion, and the freestream will have properties of the predominantly carbon dioxide $\left(\mathrm{CO}_{2}\right)$ Martian atmosphere. Clearly a part of the uncertainties in scaling is associated with the gas properties differences between the interference in the objective full-scale situation and the interference simulated in the wind tunnel. Unfortunately, with a couple of exceptions where testing included sensitivities to changing the plume gas to helium, only the air on air data is found in the literature.

The scaling model described in the Appendix, like most such models for scaling complex flows, includes the assumption that both the plume and freestream are perfect gases. As such, the thermodynamics of the mixing process modeled is transparent to differences between temperature and molecular weight. The viscosity and thermal 
conductivity are not explicitly addressed. As noted in Section II, the requirement to match Reynolds number is implied by the momentum balance used in the derivation. Both gases are assumed to remain in chemical equilibrium.

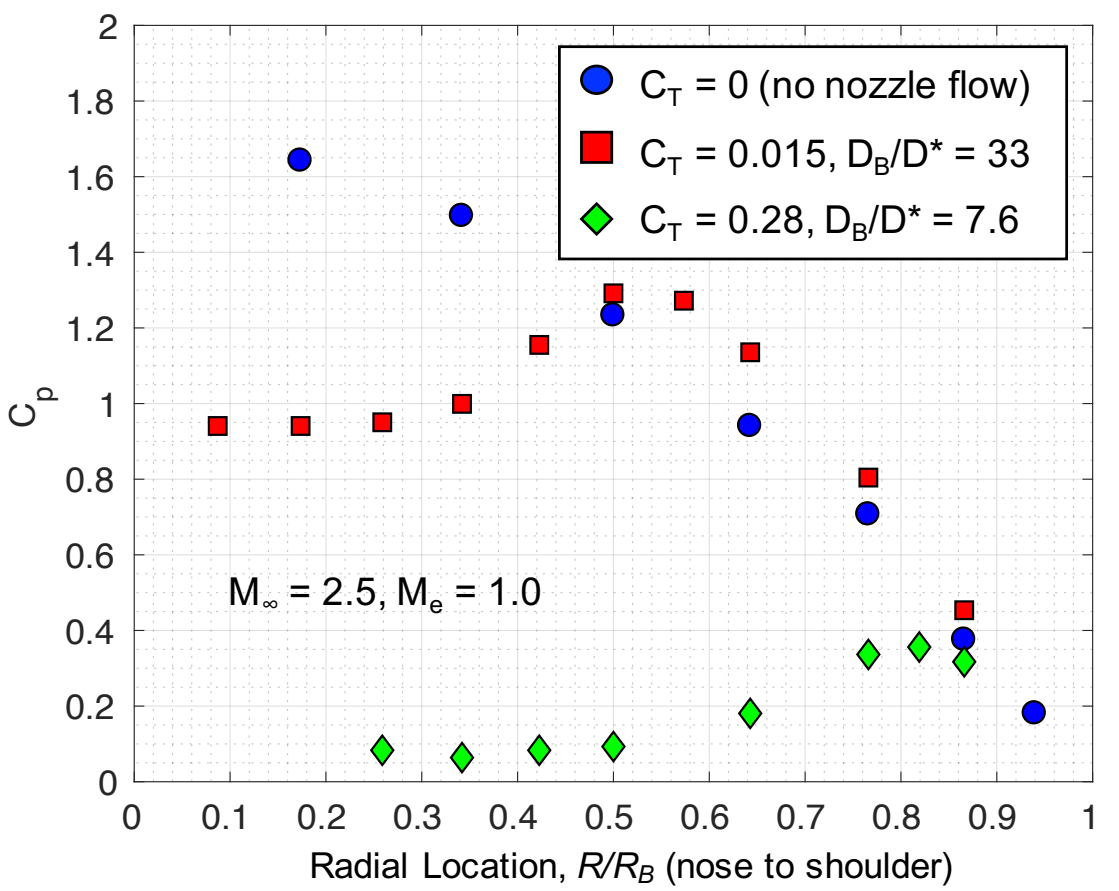

Fig. 13 Independent influence of $D_{B} / D^{*}$ on AI-induced pressure distributions.

Even if Mars-relevant gas properties can be simulated at wind tunnel and model geometry sizes, the question of scaling remains. To the extent that both plume and freestream behave as perfect gases, first principles suggest that the Mach and Reynolds numbers of both flows should be matched in ground testing. However, projected geometries for EDL at Mars are predominantly blunter bodies with diameters in excess of 15 meters, physical scales impossible to use in wind tunnels. Consequently, model sizes are likely driven to nearly two orders of magnitude smaller than the sizes of the vehicles they are to simulate. Finally, the size of the propulsion system that corresponds with the flow conditions of the wind tunnel must be specified. In general, there have been two approaches to scaling rocket propulsion systems in ground testing: either mass flow ratio or the momentum ratio between the plume and the freestream. A model for deriving the application of these approaches to scaling is described in the Appendix. The approach that is the closest approximation can only be determined through carefully constructed scaling experiments or through reliable CFD simulations designed for that purpose.

When gases other than those involved in the flight implementation of SRP are to be used in wind tunnel testing, the complexity of the scaling increases, as the requirement for selection of properties of the gases that are to be matched by the simulant plume and freestream gases must be added. Here, a scaling model, like that in the Appendix, should be the basis for the gas properties selection for either mass flow or momentum ratio scaling. In the model described in the Appendix, Eq. 14 represents the momentum ratio scaling in terms of the gas properties of the interfering flows. All of the same variables appear in this equation as have been addressed in the wind tunnel experiments involving air plumes interfering with air freestreams. Two additional parameters appear (before considerations of the higher order effects under the integrals): the ratio of ratios of specific heat between the plume and freestream gases, and the ratio of ratios of the total temperature to molecular weight. In Fig. 14, examples of these ratios for a few common gas combinations are shown, with those for typical liquid $\mathrm{O}_{2} / \mathrm{CH}_{4}$ combustion plumes. As shown in Fig. 14, this scaling model suggests that low molecular weight is interchangeable with high temperature in the relationship between the plume and freestream gases. For instance, the very low molecular weight of hydrogen $\left(\mathrm{H}_{2}\right)$ raises its equivalence to the temperature of combustion products in simulating plume gas mixtures. 


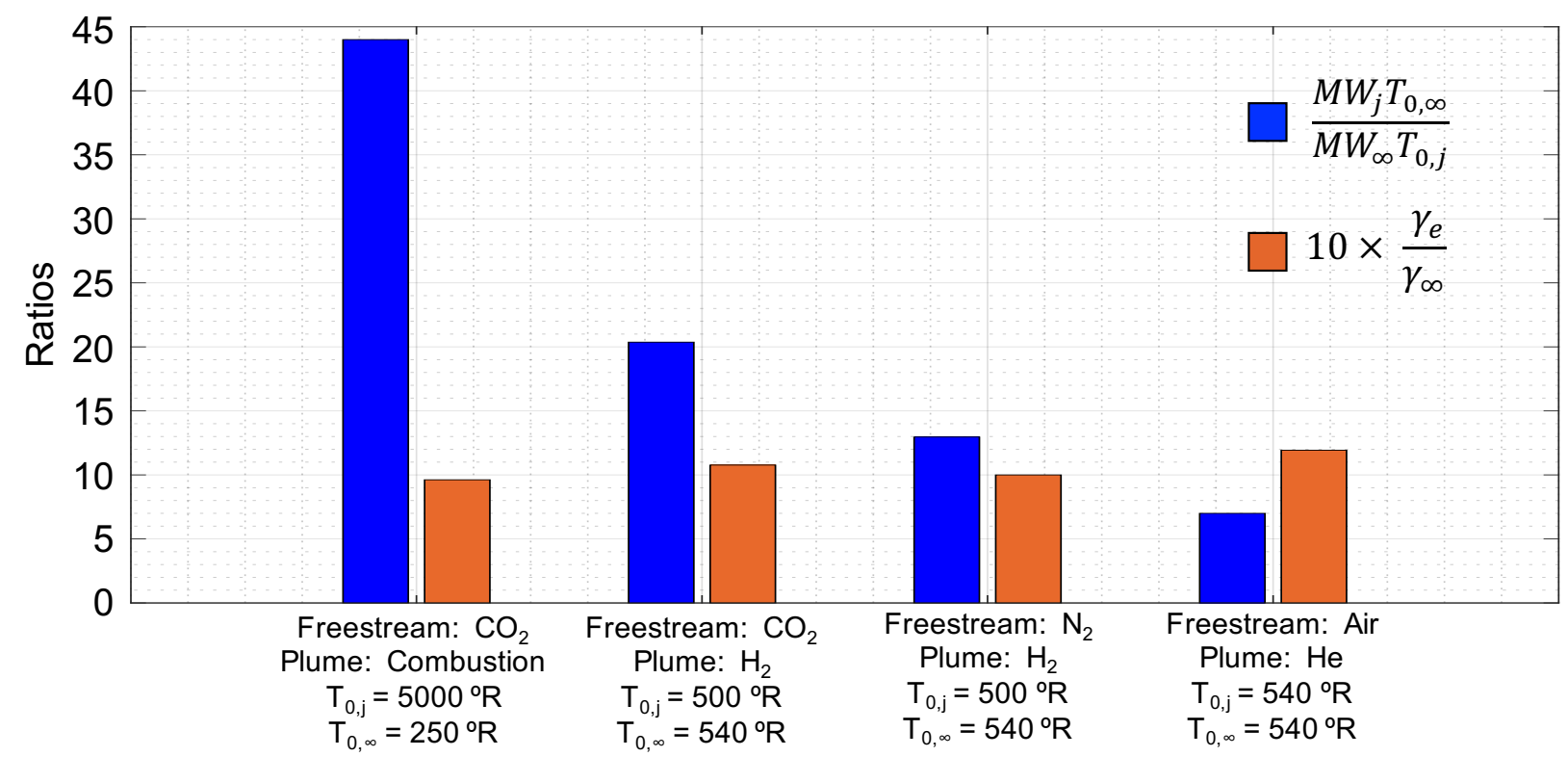

Fig. 14 Gas properties scaling parameters for representative plume and freestream gas combinations.

With few exceptions, the AI wind tunnel test database has been collected for air plumes interfering with air freestreams at approximately equal total temperatures. Consequently, data on the influence that differences in gas properties between the interfering flows are scarce. Such differences could be quite large for gases commonly used in wind tunnels to simulate plumes composed of combustion products relevant to Mars EDL. The exceptions in the literature are sensitivities of the AI to changing the plume gas from air to helium $[7,10]$. While there are arguments for varying nozzle geometry to scale air plumes with helium $(\mathrm{He})$ plumes, the selection of helium to test the sensitivity of SRP AI to variations in plume gas properties is principally a matter of convenience. Helium is readily available and compatible with most wind tunnel operations. The factor of 7 difference in molecular weight between air and helium is significant in altering the speed of sound in the plume at constant chamber temperature and exit Mach number. As indicated in the scaling equations in the Appendix, this is equivalent to a similar difference in chamber temperature. Direct comparisons of this sensitivity are complex because the ratio of specific heats for helium $(\gamma=$ 1.67) is so much different than for air $(\gamma=1.4)$. As described by Cassel et al. [13], this variable can be normalized by extending testing to a third gas (such as argon) with the same ratio of specific heats but different molecular weight than helium. No cases have been found where such has been done in SRP testing.

Following the tests developed previously in this section for scaling air plumes into air freestreams, the helium data from Romeo and Sterrett [7] are shown in Fig. 15. The disturbance size variation, with both momentum ratio and $p_{e} /$ $p_{0,2}$ for helium plumes, follows the general trends seen previously for air plumes. This testing, with helium plumes in an air freestream, used the same models and nozzles as used for the air plumes testing described above, yielding a higher exit Mach number for helium. The data reported by Jarvinen and Adams [10] for AI of air with helium plumes was not included in this testing of scaling, as there were no cases where $p_{e} / p_{0,2}$ was larger than unity.

As indicated in Table 1 and Fig. 14, the equations from the model described in the Appendix do not predict that $\mathrm{AI}$ of a helium plume into air should correlate with AI of an air plume into air. That correlation would only be expected when both the ratio of specific heats and the ratio of the chamber temperature-to-molecular weight are the same for the two plume gases. Both ratios are unity for an air plume into an air freestream (at equal chamber temperatures). For helium as the plume gas, the specific heats ratio is larger than unity, and the temperature-molecular weight ratio is a small fraction (below the recognizable scale in Fig. 14).

The correlation of shock height with momentum ratio appears to improve when comparing the helium plume from the sonic nozzle with the air plume from the supersonic nozzle than when comparing both plumes from supersonic nozzles. Fig. 15 indicates that all data for helium plumes and data for air plumes with the higher exit Mach number fail the $p_{e} / p_{0,2}$ test for the under-expanded plume mode. With either gas for the plume, spiked and erratic plume lengths occur at lower values of $p_{e} / p_{0,2}$ and follow the trend indicated in previous figures for the air-on-air AI. Because of the differences in density at constant pressure, it may be expected that the differences in disturbance scale with changing gas properties may correlate better with mass flow ratio than with momentum ratio. This correlation is illustrated in Fig. 16. Here, the differences in disturbance size between the two gases at low exit Mach number, where 
$p_{e} / p_{0,2}$ values are the highest (likely to be where the under-expanded plume mode is more stable), are more pronounced than with the momentum ratio correlation. If $p_{e} / p_{0,2}$ exceeding unity is a criterion for similitude in the interference behavior, restricting the data to be correlated to satisfy that criterion should result in correlation of plume (disturbance) size. Figure 17 illustrates this correlation.

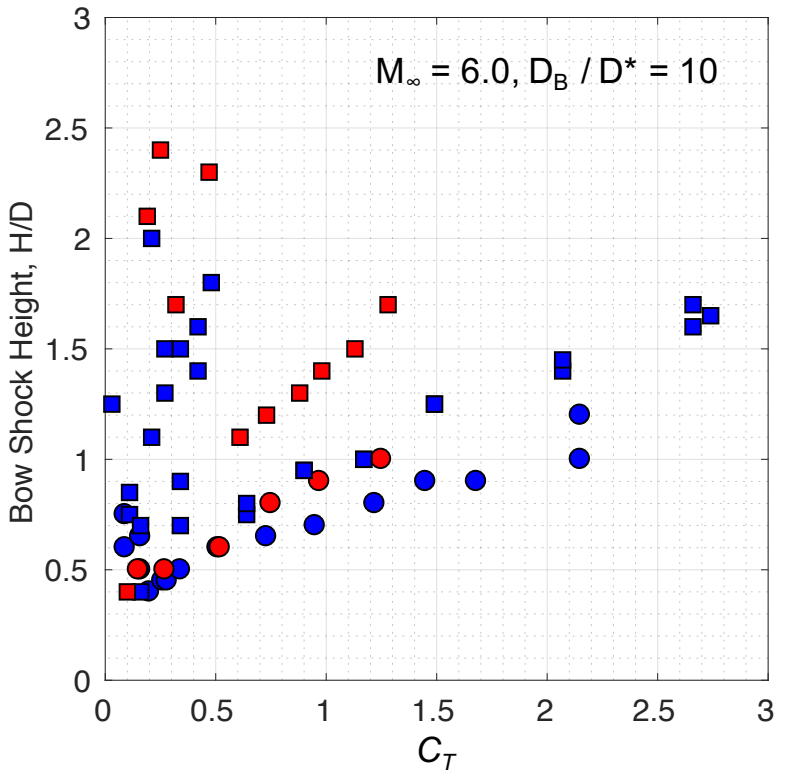

(a)

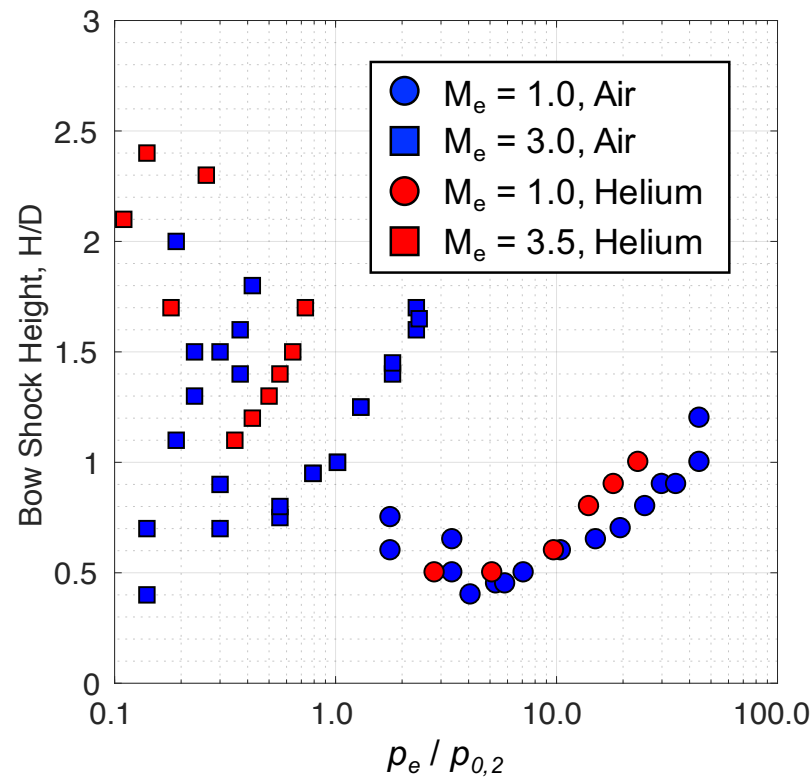

(b)

Fig. 15 Comparison of air and helium plume interference with momentum ratio and $p_{e} / p_{0,2}$.

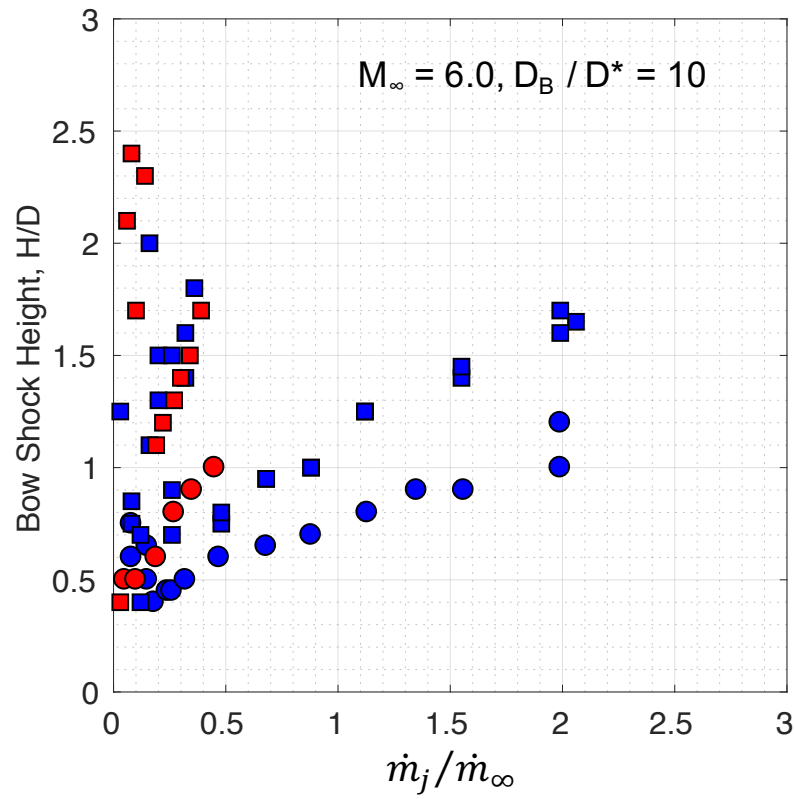

(a)

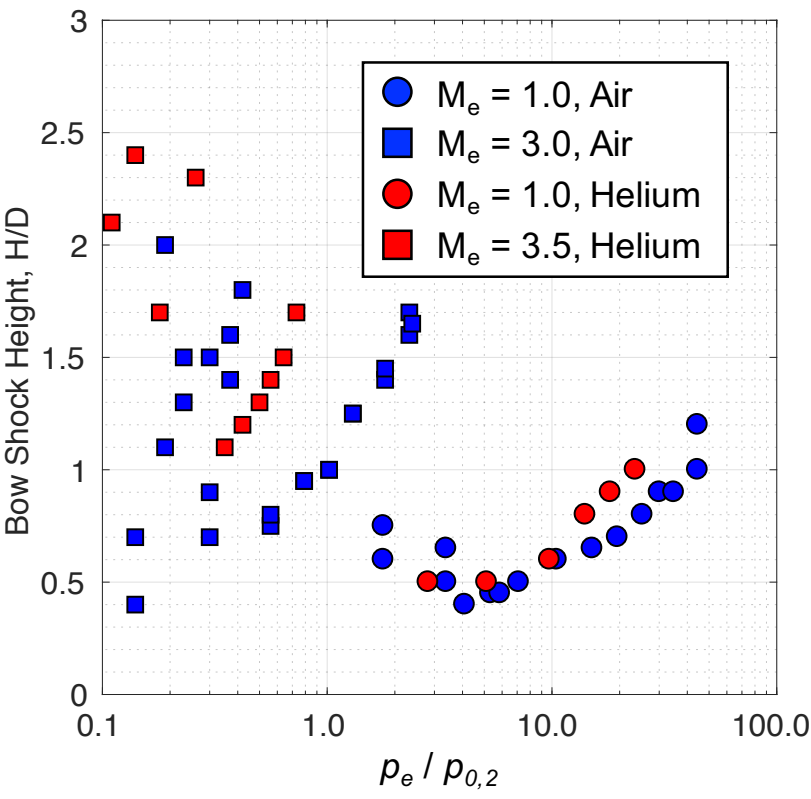

(b)

Fig. 16 Comparison of air and helium plume interference scaling with mass flow ratio at sonic and supersonic nozzle exit Mach numbers. 


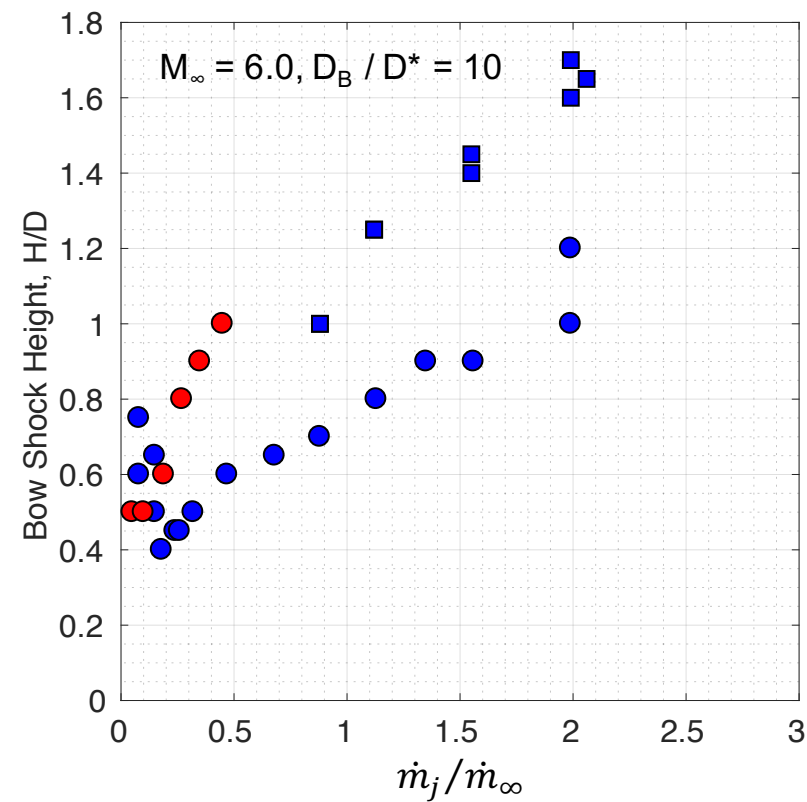

(a)

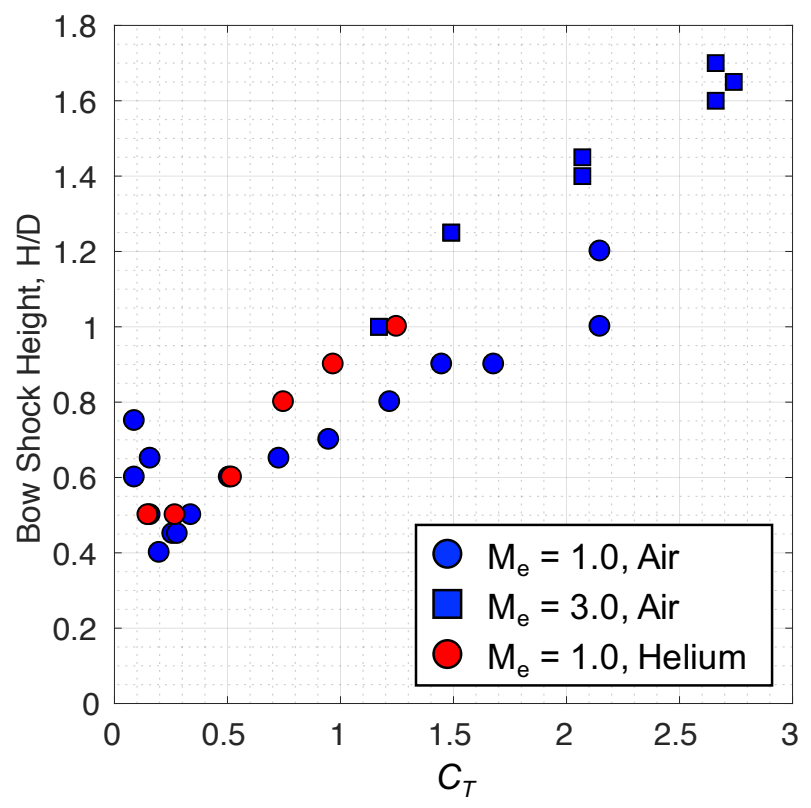

(b)

Fig. 17 Comparison of air and helium plume interference scaling with mass flow ratio for $p_{e} / p_{0,2}>1$.

With the constraint on $p_{e} / p_{0,2}$ that appears to assure overexpansion of a single plume, the correlations with both mass flow and momentum ratio are tightened. While the accuracy of the historical data is unknown, it appears that the correlations are generally improved between the data for the helium plume with $M_{e}=1$ and the air plume with $M_{e}=3$ then when the comparison is at a constant exit Mach number. For these exit Mach numbers, the mass flow and momentum ratios are approaching equality. Consequently, if one of these is a correlating parameter, changing the plume gas to another with the same relationship should also yield a similar correlation.

Higher order evidence of the AI behavior is provided by high frequency pressure measurements and schlieren images recorded in recent NASA testing by Berry et al. [4] and analyzed by Codoni and Berry [14]. Figure 18 shows high speed schlieren images of AI testing that produced the data credited to Berry et al. [4] in the preceding figures. The two images in Fig. 18 were obtained at framing rates of 5,000 frames per second. The image labelled "Single frame intensity" is the density gradient recorded in a single frame of video. The image labelled "Average intensity" is the average of gradients recorded over a large number of frames. Flowfield unsteadiness appears in the single image as a blurred region that is not apparent in the average image. The latter is closer to what would have appeared in high quality analog images and similar to those images found in the literature from historical testing.

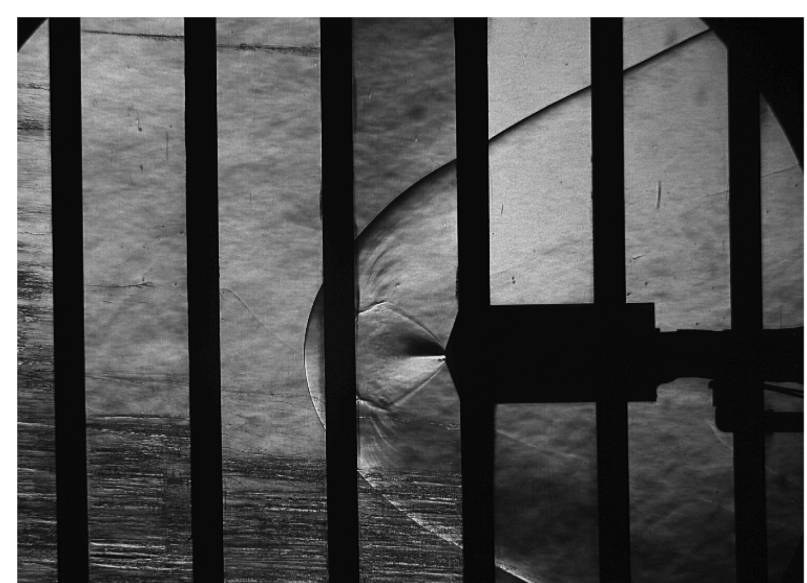

(a) Single-frame image

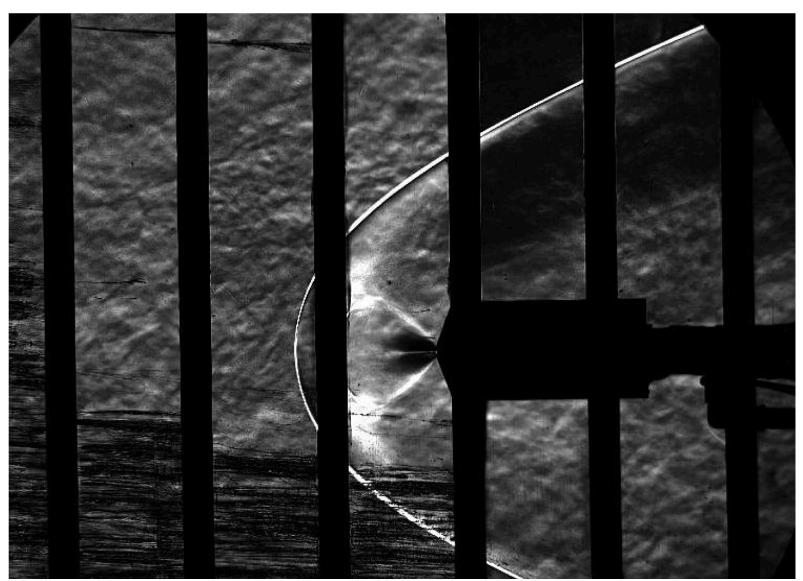

(b) Standard deviation

Fig. 18 Processed high speed schlieren images of unsteady AI $\left(M_{\infty}=4.6\right)[14]$. 
In this test, pressure fluctuations were measured by high frequency response Kulite transducers sampled at $40 \mathrm{kHz}$, and power spectral density (PSD) as a function of frequency was then calculated. Examples of these are shown in Fig. 19, from Codoni and Berry [14]. They note that the absence of spikes in the PSD for the plume-off case, in the leftside set of curves, indicates relatively steady flow. For the lower value of $C_{T}$, in the center set of curves, the pronounced spikes at frequencies near $2 \mathrm{kHz}$ indicate significant unsteadiness with fluctuations at those frequencies. For the zero angle of attack case, there are distinct harmonics at approximately $2 \mathrm{kHz}$ intervals above the primary peak. At the higher value of $C_{T}$, in the right-side curves, the unsteadiness is similar to that at the lower value of $C_{T}$, but the peaks and harmonics occur at lower frequencies for the zero angle of attack case.

As noted by Codoni and Berry [14], this shift in frequency is indicative that the scaling length for the periods associated with the peaks in unsteadiness increases with higher $C_{T}$. Since the linear dimension of the momentum ratiobased disturbance size scaling is the square root of $C_{T}$, this is also indicative of the validity of that scaling. It was further noted that " $\mathrm{kHz}$ region" unsteady flows are frequently associated with free shear layers and boundary layer separation, both of which are characteristics of the phenomena are present in the SRP AI region. The further decrease in frequency with increase in angle of attack may be indicative of coupling between the AI separated flow and incipient separation due to angle of attack. At the higher value of $C_{T}$, the intensity of the peak is higher for the same angle of attack. This may indicate that the larger disturbed region, at zero angle of attack, couples more strongly into (possibly accelerates) the insipient separation.
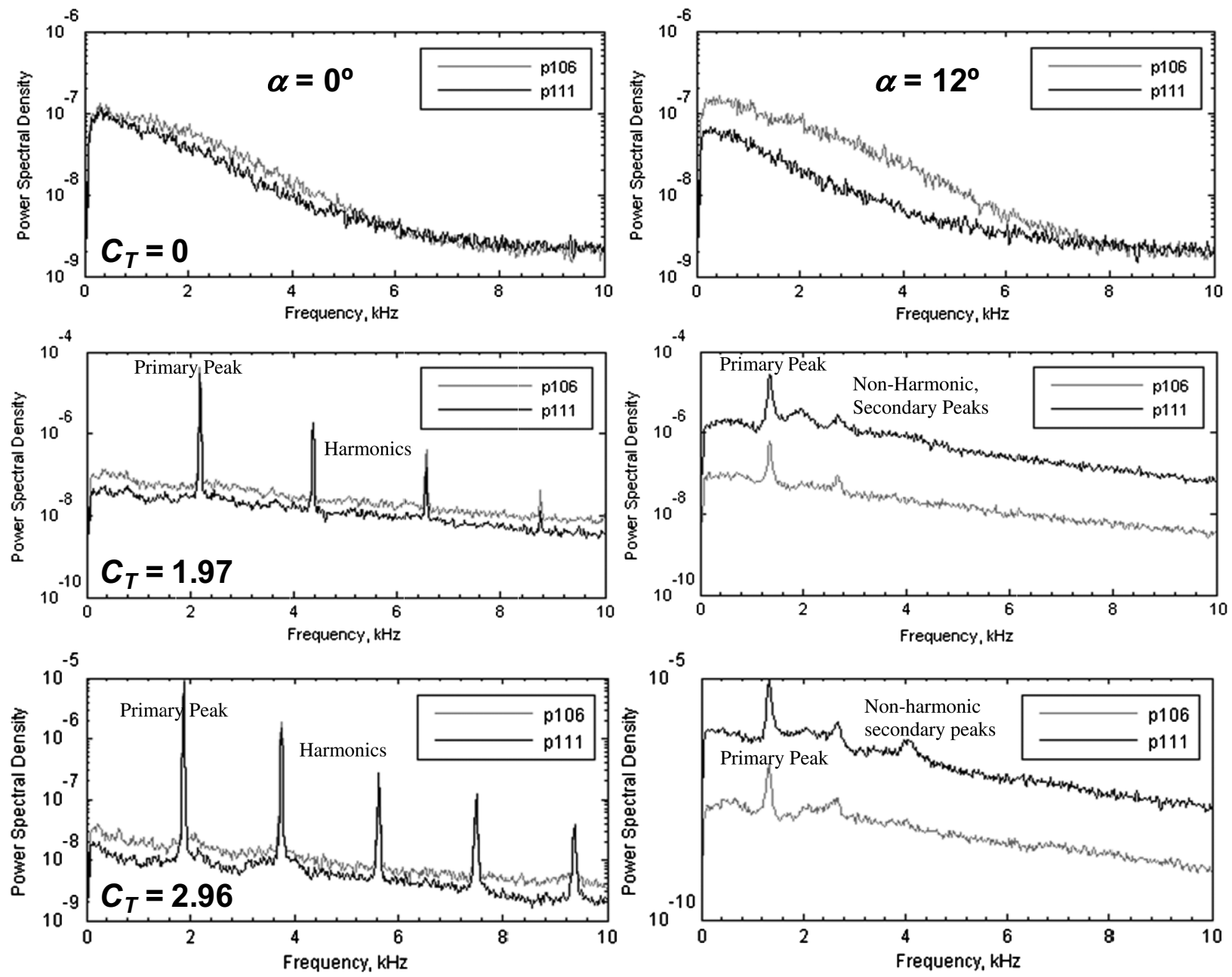

Fig. 19 Pressure fluctuation intensity for under-expanded plume AI at $\left(M_{\infty}=4.6\right)$. 


\section{Summary}

In general, wind tunnel data available in the open literature validates the following postulates: (1) Conservation of momentum-based similitude scales the size of the bow shock bounding the upstream interference region of the single nozzle supersonic retropropulsion flowfield, and (2) scaling is valid when two explicit conditions are met. The first is that the pressure at the exit of the nozzle is higher than the post-shock stagnation pressure, and the second is that the aerodynamics thrust coefficient exceeds unity. The implication of this validation is that the plume interference flowfield achieves self-similar changes in size under these conditions. The data supporting these conclusions are principally for interference between air plumes and an air freestream. However, these conclusions are also supported by limited data for helium plumes interfering with an air freestream.

A geometry similitude variable, $D_{B} / D^{*}$, implicit in the aerodynamics thrust coefficient, appears to have an independent influence when considered in extremes. At high values $\left(D_{B} / D^{*}>40\right)$ the drag momentum of the body dominates the plume interference, disrupting the behavior of the flow similitude. At low values $\left(D_{B} / D^{*}<10\right)$, the plume momentum dominates the body drag momentum. This ratio can independently influence the interference flowfield size and shape in the single nozzle limit.

The data reviewed are insufficient to test general scaling of plume gas properties, as there are insufficient data on simulation of SRP AI with one plume gas through use of a different plume gas, or a gas at a significantly different chamber temperature. Very limited data, for helium plumes exhausting into air freestreams, supports the momentum scaling conclusions under restricted circumstances. These are that the helium plume interaction correlates with the air plume interaction when only the exit Mach number of the helium plume is changed in the AI geometry and flow parameters. The exit Mach number must be selected such that the ratio of mass flow between the plumes and the freestream approach equality with the momentum ratio.

\section{Appendix - Development of Scaling Laws}

There are three approaches in practice for deriving similitude requirements for extrapolation from subscale testing. The first and most common is an engineering judgement-based combination of similitude previously derived, and proven effective, for various aspects of the flow of interest. The second is a dimensional analysis applied to postulated measurement dimensions and dependent variables describing the flow. The third is the formulation of a model of the flow and derivation of integral/differential equations describing the fluid mechanics in non-dimensional parameters. To apply any of these approaches, in cases of such complex fluid dynamics as SRP AI, the first step is to model the interacting flows at a level of description above the specific modes. The objective in each approach is the highest level of approximation that yields parameters pertinent to scaling some large, definable region or feature of the interference flow. The independent variables in the parameters must be controllable in the design of the experiment. In general, the similitude parameters combine features of the freestream and exhaust plume with the geometry of each. In this way, the experiment description is independent of dimensional scale. Most often, the range of adequate approximation must be determined experimentally.

The third of these approaches to scaling was employed for this analysis. To make this approach tractable, it was necessary to first generalize and simplify both the freestream and exhaust plume flows. Then, conservation principles were applied to the generalized interaction of the flows, and governing equations were derived in terms of the flow variables. The generalized model used here is illustrated in Fig.A.1.

There are two separate flows to be modeled: the plume and the freestream. Each flow has both fluid properties and an associated geometry. To keep the general equations manageable, the representation is defined to be an axially symmetric nozzle on the centerline of an axially symmetric vehicle at zero angle of attack. The plume, or nozzle flow, is in the direction of flight (i.e. retropropulsive). An axially symmetric control volume is constructed that bounds the AI region. The axial symmetry is not a necessary feature of the model; it is postulated to make some of the integrals in the derivation of conservation equations more tractable. The internal surface of the nozzle and the external mold line of the vehicle are both postulated to be continuous, smooth, solid surfaces.

The exhaust plume flow enters the control volume through the nozzle throat where it is uniform, sonic, and parallel to the nozzle centerline. It is bounded by the nozzle through which it expands until it encounters the freestream in the opposing direction (which occurs outside of the nozzle for a sufficiently under-expanded nozzle). At this encounter, the exhaust plume begins mixing with the opposing freestream to form the inner rhombus of a presumed axisymmetric free shear layer that flows back over the vehicle OML. 
The outer rhombus of the shear layer is formed from the freestream. It enters the control volume as a uniform supersonic flow that encounters the opposing exhaust plume, combined with the vehicle forebody. In supersonic freestream flow, the encounter results in a vehicle bow shock that bounds the outer rhombus of the mixing layer as it flows from the freestream/plume mutual stagnation point downstream of the bow shock. For a sufficiently underexpanded plume, that mutual stagnation point is also downstream in the plume (toward the bow shock) from shocks formed by the expansion of the exhaust plume. The control volume outer boundary is taken to be where the outer rhombus of the mixing layer dissipates into the undisturbed flowfield of the vehicle.

The conservation equations are formed by integrating the conservation of mass, momentum, and energy around the control volume. The process is assumed to be in an equilibrium steady state. In the analyses reported here, the scaling law applied is derived from conservation of momentum, as it has been found to be the dominant influence on disturbance size in scaling AI with reaction control plumes [15]. In principal, the few experiments with excursions in plume gas from air to helium provide an initial approximation of separating mass flow and momentum flow scaling. Consequently, both the conservation of mass and conservation of momentum scaling derivations are described here.

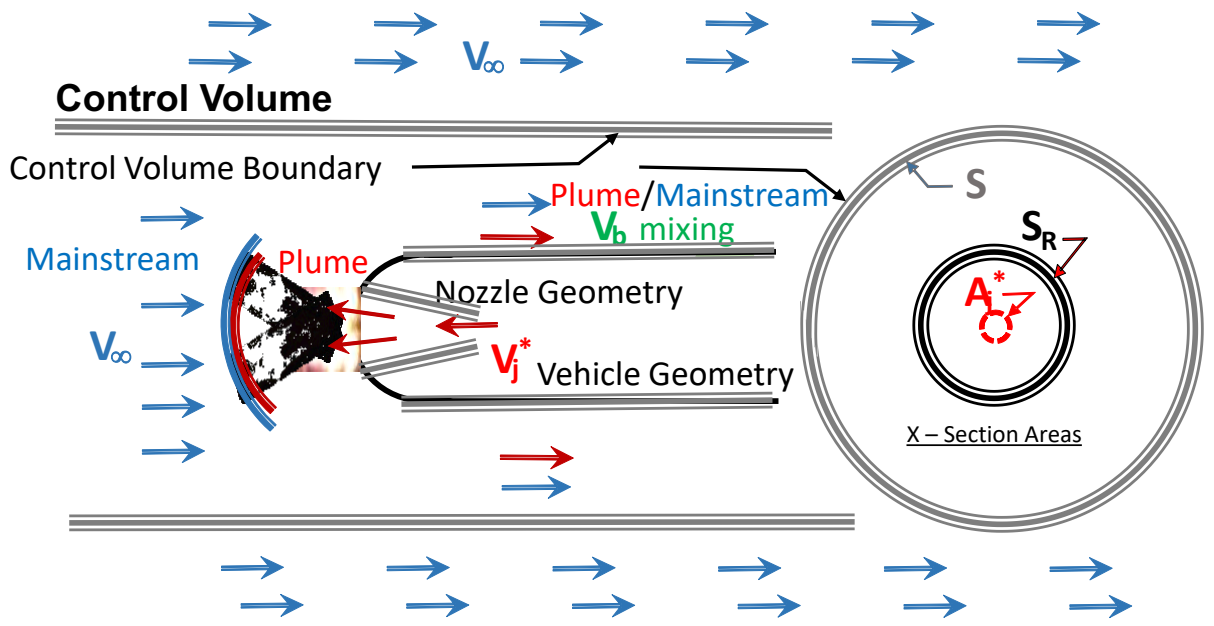

Fig. A.1 Generalized interference flow model.

Following the model above, the mass flow through the control volume can be integrated to yield:

$$
\rho_{\infty} \mathrm{V}_{\infty} \mathrm{S}+\rho_{\mathrm{j}} \mathrm{V}_{\mathrm{j}} \mathrm{A}_{\mathrm{j}}=\int_{\mathrm{S}_{\mathrm{R}}}^{\mathrm{S}} \rho_{\mathrm{b}_{\infty}(\mathrm{r})} \mathrm{V}_{\mathrm{b}} \mathrm{d} S+\int_{\mathrm{S}_{\mathrm{R}}}^{\mathrm{S}} \rho_{\mathrm{b}_{\mathrm{j}}(\mathrm{r})} \mathrm{V}_{\mathrm{b}} \mathrm{dS}
$$

When that integral is rearranged and reduced to non-dimensional form by dividing by $\rho_{\infty} \mathrm{V}_{\infty} S_{R}$, the result is:

$$
\frac{\mathrm{s}}{S_{R}}+\frac{\rho_{\mathrm{j}} \mathrm{V}_{\mathrm{j}} \mathrm{A}_{\mathrm{j}}}{\rho_{\infty} \mathrm{V}_{\infty} S_{R}}=\int_{1}^{\frac{\mathrm{s}}{S_{R}}} \frac{\left[\rho_{\mathrm{b}_{\infty}(\mathrm{r})}+\rho_{\mathrm{b}_{\mathrm{j}}(\mathrm{r})}\right]}{\rho_{\infty}} \frac{\mathrm{V}_{\mathrm{b}}}{\mathrm{V}_{\infty}} \mathrm{d}\left(\frac{\mathrm{s}}{s_{R}}\right)
$$

Returning to the general conservation of mass equation, the ratio of input mass flows appears on the left. The integral of partial fractions on the right represents the mixing layer flowing from the control volume at the base of the vehicle. Since the property distributions in the mixing layer are unknown, the integral cannot be resolved. However, the equation clearly relates the mass flow through the control volume to the ratio of the cross-sectional areas of the control volume and the vehicle.

$$
\frac{\mathrm{s}}{S_{R}}=-\frac{\rho_{\mathrm{j}} \mathrm{V}_{\mathrm{j}} \mathrm{A}_{\mathrm{j}}}{\rho_{\infty} \mathrm{V}_{\infty} S_{R}}+\int_{1}^{\frac{\mathrm{s}}{S_{R}}} \frac{\left[\rho_{\mathrm{b}_{\infty}(\mathrm{r})}+\rho_{\mathrm{b}_{\mathrm{j}}(\mathrm{r})}\right]}{\rho_{\infty}} \frac{\mathrm{V}_{\mathrm{b}}}{\mathrm{V}_{\infty}} \mathrm{d}\left(\frac{\mathrm{s}}{S_{R}}\right)
$$

Since the control volume is taken to extend to the radial extent of the SRP disturbance, the disturbance size is proportional to this ratio. To the extent that there is only one numerator and denominator set for each value of the ratio of mass flow rates, the integral on the right is a unique function of that ratio. In addition, the ratio of control volume and vehicle areas is uniquely associated with the difference of the terms on the right. Therefore, all flows that meet those conditions are self-similar, irrespective of the scale of $S_{R}$. 
However, that uniqueness is unlikely. There are certainly more than a few combinations of numerator and denominator that arrive at the same ratio. For different combinations, the distributions of flow properties at the base are likely different. Then, the integral and the ratio of control volume to vehicle areas would be different. Differences between the two flows can be easier to identify if they are described in terms of physical and thermodynamics properties.

In the physical variables of the equations above, the thermodynamics of the flowfield are unrestricted within the control volume. However, to simplify evaluation of the governing physical properties of each of the flows, it is convenient to make two idealizations. First, both the freestream and plume flows are assumed to be perfect gases. Second, the flow in the nozzle, from chamber to exit, is assumed to be isentropic. Implicit in these assumptions is the absence of friction and heat transfer.

The first assumption permits use of the perfect gas equation of state. The second assumption relegates flow in the fixed area ratio nozzle to dependence only on Mach number and the ratio of specific heats. The pertinent relationships can be written as:

$$
\begin{gathered}
p=\rho R T \\
a^{2}=\gamma R T \\
\frac{T_{o}}{T}=f_{1}(M, \gamma) \\
\frac{p_{o}}{p}=f_{2}(M, \gamma) \\
\frac{p_{t_{2}}}{p}=f_{3}(M, \gamma)
\end{gathered}
$$

Then, when these are substituted for the products of density and velocity, the equation for the disturbance size can be written as:

$\frac{\mathrm{s}}{S_{R}}=-\frac{\mathrm{A}_{\mathrm{j}}}{S_{R}} \frac{f_{2}\left(M_{e}, \gamma_{j}\right)}{f_{2}\left(M_{j}, \gamma_{j}\right)} f_{3}\left(M_{\infty}, \gamma_{\infty}\right) \frac{f_{1}\left(M_{j}, \gamma_{j}\right)}{f_{1}\left(M_{\infty}, \gamma_{\infty}\right)} \frac{p_{e}}{p_{t_{2 \infty}}} \frac{M_{j}}{M_{\infty}}\left(\frac{\gamma_{j}}{\gamma_{\infty}}\right)^{1 / 2}\left(\frac{T_{\infty \infty} / M W_{\infty}}{T_{o_{j}} / M W_{j}}\right)^{1 / 2}+\int_{1}^{\frac{\mathrm{s}}{S_{R}}} \frac{\rho_{\mathrm{b}_{\infty}(\mathrm{r})}+\rho_{\mathrm{b}_{\mathrm{j}}}(\mathrm{r})}{\rho_{\infty}} \frac{\mathrm{V}_{\mathrm{b}}(r)}{\mathrm{V}_{\infty}} \mathrm{d}\left(\frac{\mathrm{s}}{S_{R}}\right)$

It is important to note that the temperatures of the gases only appear in quotients with the molecular weights. Consequently, similitude is achieved by matching these quotients, without matching absolute values.

The integral in the conservation of mass equation is not as easy to transform. The thermodynamics properties, represented by the densities of the two species of gases, have undergone non-isentropic processes. Therefore, the stagnation pressure is no longer constant at the downstream integral. The stagnation total enthalpy is assumed constant for each species in the model described here.

The integral of the flow of momentum through the control volume, in the model in Fig. A.1, results in the following:

$$
\rho_{\infty} \mathrm{V}_{\infty}{ }^{2} \mathrm{~S}+\rho_{\mathrm{j}} \mathrm{V}_{\mathrm{j}}^{2} \mathrm{~A}_{\mathrm{j}}-\left[\int_{\mathrm{S}_{\mathrm{R}}}^{\mathrm{S}} \rho_{\mathrm{b}_{\infty}(\mathrm{r})} \mathrm{V}_{\mathrm{b}}^{2} \mathrm{~d} \mathrm{~S}+\int_{\mathrm{S}_{\mathrm{R}}}^{\mathrm{S}} \rho_{\mathrm{b}_{\mathrm{j}}(\mathrm{r})} \mathrm{V}_{\mathrm{b}}^{2} \mathrm{~d} S\right]=-F_{p}
$$

Where $F_{p}$ is the sum of the aerodynamic pressure forces (including plume back pressure). Dividing by $\rho_{\infty} \mathrm{V}_{\infty}{ }^{2} \mathrm{~S}_{R}$ yields:

$$
\frac{\mathrm{s}}{S_{R}}+\frac{\rho_{\mathrm{j}} \mathrm{V}_{\mathrm{j}}^{2} \mathrm{~A}_{\mathrm{j}}}{\rho_{\infty} \mathrm{V}_{\infty}{ }^{2} S_{R}}-\left[\int_{1}^{\frac{s}{S_{R}}} \frac{\left.\rho_{\mathrm{b}_{\infty}(\mathrm{r})}+\rho_{\mathrm{b}_{\mathrm{j}}(\mathrm{r})}\right]}{\rho_{\infty}}\left(\frac{V_{b}(r)}{V_{\infty}}\right)^{2} \mathrm{~d} \frac{\mathrm{s}}{S_{R}}\right]=-F_{p}
$$

Defining the following quantities:

$$
\begin{gathered}
q_{\infty}=\frac{\rho_{\infty} \mathrm{V}_{\infty}^{2}}{2} \\
C_{T_{v}}=\frac{\text { Vacuum Thrust }\left(T_{v}\right)}{q_{\infty} S_{R}}
\end{gathered}
$$




$$
C_{F_{p}}=\frac{\text { Pressure-induced forces }\left(F_{p}\right)}{q_{\infty} S_{R}}
$$

Then, conservation of momentum can be written as:

$$
\frac{\mathrm{s}}{S_{R}}=-\frac{1}{2}\left(C_{F_{p}}+C_{T_{v}}\right)+\left[\int_{1}^{\frac{\mathrm{s}}{S_{R}}} \frac{\left[\rho_{\mathrm{b}_{\infty}(\mathrm{r})}+\rho_{\mathrm{b}_{\mathrm{j}}(\mathrm{r})}\right]}{\rho_{\infty}}\left(\frac{V_{b}}{V_{\infty}}\right)^{2} \mathrm{~d} \frac{\mathrm{s}}{S_{R}}\right]
$$

The same transformation used to convert the mass flow ratio in the mass conservation equation can be used to convert the incoming momentum flow to thermodynamics quantities. To substitute this ratio in the conservation of momentum equation, the aerodynamics thrust coefficient is written in explicit variables, and the equation is rearranged:

$$
\frac{\mathrm{s}}{S_{R}}=-\left\{\frac{\rho_{\mathrm{j}} \mathrm{V}_{\mathrm{j}}^{2} \mathrm{~A}_{\mathrm{j}}}{\rho_{\infty} \mathrm{V}_{\infty}^{2} S_{R}}-\left[\int_{1}^{\frac{\mathrm{s}}{S_{R}}} \frac{\left[\rho_{\mathrm{b}_{\infty}(\mathrm{r})}+\rho_{\mathrm{b}_{\mathrm{j}}(\mathrm{r})}\right]}{\rho_{\infty}}\left(\frac{V_{b}}{V_{\infty}}\right)^{2} \mathrm{~d} \frac{\mathrm{s}}{S_{R}}\right]+\frac{1}{2} C_{F_{p}}\right\}
$$

Then the transformed equation can be written as:

$\frac{\mathrm{s}}{S_{R}}=-\left\{\frac{A_{j}}{S_{R}}\left[\frac{f_{2}\left(M_{e}, \gamma_{j}\right)}{f_{2}\left(M_{j}, \gamma_{j}\right)} f_{3}\left(M_{\infty}, \gamma_{\infty}\right) \frac{f_{1}\left(M_{j}, \gamma_{j}\right)}{f_{1}\left(M_{\infty}, \gamma_{\infty}\right)} \frac{p_{e}}{p_{t_{2_{\infty}}}}\left(\frac{M_{j}}{M_{\infty}}\right)^{2} \frac{\gamma_{j}}{\gamma_{\infty}}\left(\frac{T_{o_{\infty}} / \boldsymbol{m}_{\infty}}{T_{o_{j}} / \boldsymbol{m}_{j}}\right)^{1 / 2}\right]-\left[\int_{1}^{\frac{\mathrm{s}}{S_{R}}} \frac{\left[\rho_{\mathrm{b}_{\infty}(\mathrm{r})}+\rho_{\mathrm{b}_{\mathrm{j}}(\mathrm{r})}\right]}{\rho_{\infty}}\left(\frac{V_{b}(r)}{V_{\infty}}\right)^{2} \mathrm{~d} \frac{\mathrm{s}}{S_{R}}\right]+\frac{1}{2} C_{F_{p}}\right\}$

In some wind tunnel testing reported to date it has been implicit that the aerodynamics thrust coefficient is an interaction flowfield size scaling parameter. If true, this would indicate that the conservation of momentum equation (Eq. 11) is dominant in scaling disturbance size. In this case, the size of the disturbance (the control volume crosssection $\left(S_{R}\right)$ in Fig. A.1, should scale with the size of the model cross-section. However, it should only be valid when the other terms in the equation are satisfied. In particular, when the geometry scale is changed, the functions that govern the coefficients representing aerodynamics on the OML in the freestream remain the same to hold $C_{F p}$ constant. Even then, the approximation is only to the order that the distributions on the right-hand side of the equations remain unchanged. Measuring the size of the disturbance is relatively straightforward, through dimensionless mapping of the displacement of the center of the bow shock. Measuring the distributions on the right-hand side, by contrast, is arduous and costly. This assumption remains an approximation in the analyses reported here.

The implication of the same equation in physical variables form, Eq. 14 below, is that the gases must be selected to match the properties that determine the functions above in that equation. Similar scaling would be implied for one of the other conservation variables if the parameters in the conservation of mass or conservation of energy equations were found to scale gross features of the flow in test data.

$\frac{\mathrm{s}}{S_{R}}=-\left\{\frac{A_{j}}{S_{R}}\left[\frac{f_{2}\left(M_{e}, \gamma_{j}\right)}{f_{2}\left(M_{j}, \gamma_{j}\right)} f_{3}\left(M_{\infty}, \gamma_{\infty}\right) \frac{f_{1}\left(M_{j}, \gamma_{j}\right)}{f_{1}\left(M_{\infty}, \gamma_{\infty}\right)} \frac{p_{e}}{p_{t_{2}}}\left(\frac{M_{j}}{M_{\infty}}\right)^{2} \frac{\gamma_{j}}{\gamma_{\infty}}\left(\frac{T_{o_{\infty}} / \boldsymbol{M} \boldsymbol{W}_{\infty}}{T_{o_{j}} / \boldsymbol{M}_{j}}\right)^{1 / 2}\right]-\left[\int_{1}^{\frac{\mathrm{s}}{S_{R}}} \frac{\left[\rho_{\mathrm{b}_{\infty}(\mathrm{r})}+\rho_{\mathrm{b}_{\mathrm{j}}(\mathrm{r})}\right]}{\rho_{\infty}}\left(\frac{V_{b}(r)}{V_{\infty}}\right)^{2} \mathrm{~d} \frac{\mathrm{s}}{S_{R}}\right]+\right.$ $\left.\frac{1}{2} C_{F_{p}}\right\}$

To formulate an algebraically simplified equation describing the conservation of energy, assumptions are added to the flow model illustrated in Fig. A-1. The freestream and plume flow entering the SRP AI are assumed to mix into a perfect gas with two components during the AI process. Here, the freestream and plume have passed through the process in a very large reservoir where they are mixed homogeneously. The resulting gas mixture flows from the reservoir through a downstream AI region unchanged from Fig. A-1.

For this simplified flow process, the conservation of energy per unit mass can be written as:

$$
h_{\infty}+\frac{v_{\infty}^{2}}{2}+h_{j}+\frac{v_{j}^{2}}{2}=h_{b_{\infty}}+h_{b_{\infty}}+\frac{v_{b}^{2}}{2}
$$


Here, the downstream enthalpy is divided between the two constituents of the assumed mixed flow. The flow of the mass carrying this energy is described by the conservation of mass equation in Eq. 3. When the energy per unit mass equation is integrated with that describing conservation of mass, the energy flow into the reservoir is:

$$
\left(\rho_{\infty} V_{\infty} S\right)\left(h_{\infty}+\frac{V_{\infty}^{2}}{2}\right)+\left(\rho_{\mathrm{j}} \mathrm{V}_{\mathrm{j}} \mathrm{A}_{\mathrm{j}}\right)\left(h_{j}+\frac{V_{j}^{2}}{2}\right)
$$

where the energy of each incoming stream is assumed uniformly distributed at its inlet. The flow of energy from the reservoir/process, through the downstream tube, is:

$$
\int_{S_{R}}^{S}\left[\rho_{\mathrm{b}_{\infty}(\mathrm{r})} \mathrm{h}_{\mathrm{b}_{\infty}(\mathrm{r})}+\rho_{\mathrm{b}_{\mathrm{j}}(\mathrm{r})} \mathrm{h}_{\mathrm{b}_{\mathrm{j}}(\mathrm{r})}\right] V_{2}(r) d S+\int_{S}^{S_{R}}\left[\rho_{\mathrm{b}_{\infty}(\mathrm{r})}+\rho_{\mathrm{b}_{\mathrm{j}}(\mathrm{r})}\right] \frac{V_{b}^{3}(r)}{2} d S
$$

where the mixture of freestream and plume flows, from the reservoir/process, is made up of the two homogenously distributed species. Then, the conservation of energy through the AI system is described by equating the rate flowing out to the rate flowing in as:

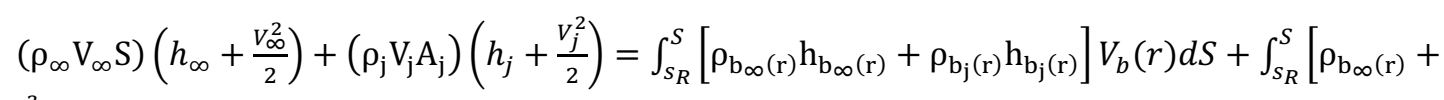

$\left.\rho_{\mathrm{b}_{\mathrm{j}}(\mathrm{r})}\right] \frac{V_{b}^{3}(r)}{2} d S$

Transforming the conservation of energy equation to physical properties is simplified by first extending the perfect gas assumption to calorically perfect. Then,

$$
h+\frac{V^{2}}{2}=h_{o}
$$

defines the stagnation enthalpy per unit mass and the equation becomes:

$$
\left(\rho_{\infty} \mathrm{V}_{\infty} S\right) h_{o_{\infty}}+\left(\rho_{\mathrm{j}} \mathrm{V}_{\mathrm{j}} \mathrm{A}_{\mathrm{j}}\right) h_{o_{j}}=\int_{S_{R}}^{S}\left[\rho_{\mathrm{b}_{\infty}(\mathrm{r})} \mathrm{h}_{\mathrm{b}_{\infty}(\mathrm{r})}+\rho_{\mathrm{b}_{\mathrm{j}}(\mathrm{r})} \mathrm{h}_{\mathrm{b}_{\mathrm{j}}(\mathrm{r})}\right] V_{b}(r) d S+\int_{S_{R}}^{S}\left[\rho_{\mathrm{b}_{\infty}(\mathrm{r})}+\rho_{\mathrm{b}_{\mathrm{j}}(\mathrm{r})}\right] \frac{V_{b}^{3}(r)}{2} d S
$$

In this form, the equation is non-dimensionalized by $\rho_{\infty} \mathrm{V}_{\infty} S_{R} h_{o_{\infty}}$ to yield:

$$
\frac{S}{S_{R}}+\frac{\left(\rho_{\mathrm{j}} \mathrm{V}_{\mathrm{j}} \mathrm{A}_{\mathrm{j}}\right) h_{o_{j}}}{\rho_{\infty} \mathrm{V}_{\infty} S_{R} h_{\infty}}=\int_{S_{R}}^{S}\left[\frac{\rho_{\mathrm{b}_{\infty}(\mathrm{r})} \mathrm{h}_{\mathrm{b}_{\infty}(\mathrm{r})}+\rho_{\mathrm{b}_{\mathrm{j}}(\mathrm{r})} \mathrm{h}_{\mathrm{b}_{\mathrm{j}}(\mathrm{r})}}{\rho_{\infty} \mathrm{V}_{\infty} S_{R} h_{o_{\infty}}}\right] V_{b}(r) d S+\int_{S_{R}}^{S} \frac{\left[\rho_{\mathrm{b}_{\infty}(\mathrm{r})}+\rho_{\mathrm{b}_{\mathrm{j}}(\mathrm{r})}\right.}{\rho_{\infty} \mathrm{V}_{\infty} S_{R} h_{o_{\infty}}} \frac{V_{b}^{3}(r)}{2} d S
$$

The right-hand side can be written in the form of the mass and momentum conservation equations as:

$$
\frac{s}{S_{R}}+\frac{\left(\rho_{\mathrm{j}} \mathrm{V}_{\mathrm{j}} \mathrm{A}_{\mathrm{j}}\right) h_{o_{j}}}{\rho_{\infty} \mathrm{V}_{\infty} S_{R} h_{o_{\infty}}}=\int_{1}^{S}\left[\frac{\rho_{\mathrm{b}_{\infty}(\mathrm{r})} \mathrm{h}_{\mathrm{b}_{\infty}(\mathrm{r})}+\rho_{\mathrm{b}_{\mathrm{j}}(\mathrm{r})} \mathrm{h}_{\mathrm{b}_{\mathrm{j}}(\mathrm{r})}}{\rho_{\infty} h_{o_{\infty}}}\right] \frac{V_{b}(r)}{\mathrm{V}_{\infty}} d \frac{s}{S_{R}}+\int_{1}^{S} \frac{\left[\rho_{\mathrm{b}_{\infty}(\mathrm{r})}+\rho_{\mathrm{b}_{\mathrm{j}}(\mathrm{r})}\right]}{\rho_{\infty}} \frac{V_{b}^{3}(r)}{2 \mathrm{~V}_{\infty} h_{o_{\infty}}} d \frac{s}{s_{R}}
$$

Further identification of convenient physical properties is facilitated by the assumption of calorically perfect gases perfect (constant ratios of specific heats). Then the total enthalpy per unit mass can be written $h_{o}=c_{p} T_{o}$ and the energy equation becomes:

$$
\frac{s}{S_{R}}+\frac{\left(\rho_{\mathrm{j}} \mathrm{V}_{\mathrm{j}} \mathrm{A}_{\mathrm{j}}\right) c_{p_{j}} T_{o_{j}}}{\rho_{\infty} \mathrm{V}_{\infty} S_{R} c_{p_{\infty} T_{\infty}}}=\int_{1}^{S}\left[\frac{{ }^{\mathrm{p}_{\infty}} \rho_{\mathrm{b}_{\infty}(\mathrm{r})}+c_{\mathrm{p}_{\mathrm{j}}} \rho_{\mathrm{b}_{\mathrm{j}}}(\mathrm{r})}{\rho_{\infty} c_{p_{\infty}}}\right] \frac{T_{b}}{T_{o_{\infty}}} \frac{V_{b}(r)}{\mathrm{V}_{\infty}} d \frac{s}{S_{R}}+\int_{1}^{S} \frac{\left[\rho_{\mathrm{b}_{\infty}(\mathrm{r})}+\rho_{\mathrm{b}_{\mathrm{j}}}(\mathrm{r})\right]}{\rho_{\infty}} \frac{V_{b}^{3}(r)}{2 \mathrm{~V}_{\infty} c_{p_{\infty} T_{\infty}}} d \frac{s}{s_{R}}
$$

As with the equations for conservation of mass and momentum, the ratio of products of density and velocity on the left-hand side can be replaced by the equivalent physical properties to yield: 


$$
\begin{aligned}
& \frac{s}{S_{R}}+\left[\frac{f_{2}\left(M_{e}, \gamma_{j}\right)}{f_{2}\left(M_{j}, \gamma_{j}\right)} f_{3}\left(M_{\infty}, \gamma_{\infty}\right) \frac{f_{1}\left(M_{j}, \gamma_{j}\right)}{f_{1}\left(M_{\infty}, \gamma_{\infty}\right)} \frac{p_{e}}{p_{t_{\infty}}} \frac{M_{j}}{M_{\infty}}\left(\frac{\gamma_{j}}{\gamma_{\infty}}\right)^{1 / 2}\left(\frac{T_{o_{\infty}} / M W_{\infty}}{T_{o_{j}} / M W_{j}}\right)^{1 / 2}\right] \frac{A_{j} c_{p_{j}} T_{o_{j}}}{S_{R} c_{p_{\infty} T_{o_{\infty}}}}= \\
& \int_{1}^{S}\left[\frac{{ }^{\mathrm{p}_{\infty}} \rho_{\mathrm{b}_{\infty}(\mathrm{r})}+\mathrm{c}_{\mathrm{p}_{\mathrm{j}}} \rho_{\mathrm{b}_{\mathrm{j}}(\mathrm{r})}}{\rho_{\infty} c_{p_{\infty}}}\right] \frac{T_{b}}{T_{\infty_{\infty}}} \frac{V_{b}(r)}{\mathrm{V}_{\infty}} d \frac{s}{S_{R}}+\int_{1}^{S} \frac{\left[\rho_{\mathrm{b}_{\infty}(\mathrm{r})}+\rho_{\mathrm{b}_{\mathrm{j}}(\mathrm{r})}\right]}{\rho_{\infty}} \frac{V_{b}^{3}(r)}{2 \mathrm{~V}_{\infty} c_{p_{\infty}} T_{o_{\infty}}} d \frac{s}{s_{R}}
\end{aligned}
$$

Or, identifying the total enthalpy and rearranging to leave the normalized disturbance area on the left-hand side:

$$
\begin{gathered}
\frac{s}{S_{R}}=-\left[\frac{f_{2}\left(M_{e}, \gamma_{j}\right)}{f_{2}\left(M_{j}, \gamma_{j}\right)} f_{3}\left(M_{\infty}, \gamma_{\infty}\right) \frac{f_{1}\left(M_{j}, \gamma_{j}\right)}{f_{1}\left(M_{\infty}, \gamma_{\infty}\right)} \frac{p_{e}}{p_{t_{2_{\infty}}}} \frac{M_{j}}{M_{\infty}}\left(\frac{\gamma_{j}}{\gamma_{\infty}}\right)^{1 / 2}\left(\frac{T_{o_{\infty}} / M \boldsymbol{W}_{\infty}}{T_{o_{j}} / \boldsymbol{M} \boldsymbol{W}_{j}}\right)^{1 / 2}\right] \frac{A_{j} h_{o_{j}}}{S_{R} h_{o_{\infty}}}+ \\
\int_{1}^{S}\left[\frac{\mathrm{p}_{\infty} \rho_{\mathrm{b}_{\infty}(\mathrm{r})}+c_{\mathrm{p}_{\mathrm{j}}} \rho_{\mathrm{b}_{\mathrm{j}}(\mathrm{r})}}{\rho_{\infty} c_{p_{\infty}}}\right] \frac{T_{b}}{T_{o_{\infty}}} \frac{V_{b}(r)}{\mathrm{V}_{\infty}} d \frac{S}{S_{R}}+\int_{1}^{S} \frac{\left[\rho_{\mathrm{b}_{\infty}(\mathrm{r})}+\rho_{\mathrm{b}_{\mathrm{j}}(\mathrm{r})}\right]}{\rho_{\infty}} \frac{V_{b}^{3}(r)}{2 \mathrm{~V}_{\infty} c_{p_{\infty} T_{\infty_{\infty}}}} d \frac{S}{s_{R}}
\end{gathered}
$$

\section{Acknowledgments}

This work has been sponsored by the NASA Space Technology Mission Directorate's Descent Systems Studies Project.

\section{References}

[1] Korzun, A. M., Braun, R. D., and Cruz, J. R., "Survey of Supersonic Retropropulsion Technology for Mars Entry, Descent, and Landing," Journal of Spacecraft and Rockets, Vol. 46, No. 5, 2009, pp. 929-937. doi:10.2514/1.41161.

[2] Dwyer Cianciolo, A. M., and Polsgrove, T. T., "Human Mars Entry, Descent, and Landing Architecture Study Overview," AIAA Paper 2016-5494, 2016.

[3] Dwyer Cianciolo, A. M., et al., "Human Mars Entry, Descent, and Landing Architecture Study: Phase 3 Summary," Accepted to AIAA SciTech2020 Conference, AIAA Paper 2020-XXXX, 2020.

[4] Berry, S. A., Rhode, M. N., and Edquist, K. T., "Supersonic Retropropulsion Validation Experiment in the NASA Langley Unitary Plan Wind Tunnel," Journal of Spacecraft and Rockets, Vol. 51, No. 3, 2014, pp. 664-679. doi:10.2514/1.A32649.

[5] Berry, S. A., Rhode, M. N., and Edquist, K. T., "Supersonic Retropropulsion Experimental Results from NASA Ames 9 x 7 Foot Supersonic Wind Tunnel,” Journal of Spacecraft and Rockets, Vol. 51, No. 3, 2014, pp. 724-734. doi:10.2514/1.A32650.

[6] Edquist, K. T., Korzun, A. M., Bibb, K. L., Schauerhamer, D. G., Ma, E. C., McCloud, P. L., Palmer, G. E., and Monk, J. D., "Comparison of Navier-Stokes Flow Solvers to Falcon 9 Supersonic Retropropulsion Flight Data," AIAA Paper 2017-5296, 2017.

[7] Romeo, D. J., and Sterrett, J. R., "Exploratory Investigation of the Effect of a Forward-Facing Jet on the Bow Shock of a Blunt Body in a Mach Number 6 Free Stream," NASA TN D-1605, Feb. 1963.

[8] McGhee, R. J., "Effects of a Retronozzle Located at the Apex of a 140-deg Blunt Cone at Mach Numbers of 3.00, 4.50, and 6.00, , NASA TN D-6002, Jan. 1971.

[9] Finley, P. J., "The Flow of a Jet from a Body Opposing a Supersonic Free Stream,” Journal of Fluid Mechanics, Vol. 26, Part 2, 1966, pp.337-368.

[10] Jarvinen, P. O., and Adams, R. H., "The Aerodynamic Characteristics of Large Angled Cones with Retrorockets," NASA CR NAS 7-576, Feb. 1970.

[11] Daso, E. O., Pritchett, V. E., Wang, T. S., Ota, D. K., Blankson, I. M., and Auslender, A. H., "Dynamics of Shock Dispersion and Interactions in Supersonic Freestreams with Counterflowing Jets," AIAA Journal, Vol. 47, No. 6, 2009, pp. 1313-1326.

[12] Bogdonoff, S. M., Vas, I. E., “Some Experiments on Hypersonic Flows,” Journal of the American Rocket Society, Vol. 32, No. 10, 1962.

[13] Cassel, L. A., Shivananda, T., Feiz, H., Kovacic, S., and Douglas, R., "Reverse Flow Interference Wind Tunnel Testing for CEV Launch Abort System (LAS)," AIAA Paper 2008-347, 2008.

[14] Codoni, J. R., and Berry, S. A., "Supersonic Retropropulsion Dynamic Data Analysis from NASA Langley Unitary Plan Wind Tunnel," Journal of Spacecraft and Rockets, Vol. 51, No. 3, 2014, pp. 715-723. doi:10.2514/1.A32619.

[15] Cassel, L.A., “Applying Jet Interaction Technology,” Journal of Spacecraft and Rockets, Vol. 40, No. 4, 2003 , pp. $523-537$. 